\title{
Are we missing the Institute of Medicine's mark? A systematic review of patient-reported outcome measures assessing quality of patient-centred cancer care
}

Flora Tzelepis*, Shiho K Rose, Robert W Sanson-Fisher, Tara Clinton-McHarg, Mariko L Carey and Christine L Paul

\begin{abstract}
Background: The Institute of Medicine (IOM) has endorsed six dimensions of patient-centredness as crucial to providing quality healthcare. These dimensions outline that care must be: 1) respectful to patients' values, preferences, and expressed needs; 2) coordinated and integrated; 3) provide information, communication, and education; 4) ensure physical comfort; 5) provide emotional support-relieving fear and anxiety; and 6) involve family and friends. However, whether patient-reported outcome measures (PROMs) comprehensively cover these dimensions remains unexplored. This systematic review examined whether PROMs designed to assess the quality of patient-centred cancer care addressed all six IOM dimensions of patient-centred care and the psychometric properties of these measures.

Methods: Medline, PsycINFO, Current Contents, Embase, CINAHL and Scopus were searched to retrieve published studies describing the development and psychometric properties of PROMs assessing the quality of patient-centred cancer care. Two authors determined if eligible PROMs included the six IOM dimensions of patient-centred care and evaluated the adequacy of psychometric properties based on recommended criteria for internal consistency, test-retest reliability, face/content validity, construct validity and cross-cultural adaptation.
\end{abstract}

Results: Across all 21 PROMs, the most commonly included IOM dimension of patient-centred care was "information, communication and education" (19 measures). In contrast, only five measures assessed the "involvement of family and friends." Two measures included one IOM-endorsed patient-centred care dimension, two measures had two dimensions, seven measures had three dimensions, five measures had four dimensions, and four measures had five dimensions. One measure, the Indicators (Non-small Cell Lung Cancer), covered all six IOM dimensions of patient-centred care, but had adequate face/content validity only. Eighteen measures met the recommended adequacy criteria for construct validity, 15 for face/content validity, seven for internal consistency, three for cross-cultural adaptation and no measure for test-retest reliability.

Conclusions: There are no psychometrically rigorous PROMs developed with cancer patients that capture all six IOM dimensions of patient-centred care. Using more than one measure or expanding existing measures to cover all six patient-centred care dimensions could improve assessment and delivery of patient-centred care. Construction of new comprehensive measures with acceptable psychometric properties that can be used with the general cancer population may also be warranted.

Keywords: Patient-centred, Quality of care, Patient-reported outcome measures, Cancer, Reliability, Validity, Systematic review

\footnotetext{
* Correspondence: Flora.Tzelepis@newcastle.edu.au

Health Behaviour Research Group, Priority Research Centre for Health

Behaviour, University of Newcastle \& Hunter Medical Research Institute,

Newcastle, New South Wales, Australia
} 


\section{Background}

The Institute of Medicine has defined high quality health care as the provision of appropriate services in a technically competent manner, and includes good communication, shared decision-making and is consistent with patient values and preferences [1]. Optimizing the structure (e.g., hospital resources, number of staff), processes (e.g., interactions between health care providers and patients, use of effective therapies) and outcomes (e.g., survival, quality of life) of health care services are crucial to achieving high quality care [1]. In 2001, the IOM published "Crossing the Quality Chasm" a broad framework which recommended improvements to the following six areas of healthcare in order to achieve high quality care: safety; effectiveness; timeliness; efficiency; equity; and patient-centredness [1]. Within the area of patientcentredness, the IOM also endorsed Gerteis et al's six dimensions of patient-centred care [2] which state that care must be: 1) respectful to patients' values, preferences, and expressed needs; 2) coordinated and integrated; 3) provide information, communication, and education; 4) ensure physical comfort; 5) provide emotional support-relieving fear and anxiety; and 6) involve family and friends [1]. The IOM's recognition of patient-centredness as an indicator of quality acknowledges the adoption of a whole-person orientation to healthcare that goes beyond solely focusing on treatment of the disease.

A variety of sources have been used to assess the quality of care that patients receive including administrative databases, cancer registries, medical records, patient self-reported measures, physician surveys, and pharmacy and laboratory data [3]. However unlike other aspects of quality, such as efficiency, patient self-report is arguably the only way to assess constructs that relate to patientcentredness. For instance, the severity of cancer pain and levels of fatigue experienced by a patient can only reliably be assessed by the patient themselves, and self-report is widely recognised as the gold standard for such assessments [4]. The value of obtaining patient self-report data is further demonstrated by research reporting that patients' perceptions of quality of health care have been associated with important medical and psychological outcomes, including quality of life [5-8], anxiety and depression [6-9]. Patients' perceptions of quality of care have also been associated with factors that directly affect the effectiveness and efficiency of health care such as the under-utilisation of treatments [10-12] and mistrust of the medical system [13,14].

Patient-reported outcome measures (PROMs) that have been designed to assess the quality of patientcentred care include measures of: 1) satisfaction with care; and 2) experiences of care. Satisfaction with care measures investigate the extent to which an individual's health care experiences met his/her expectations [15]. However, a range of factors unrelated to the actual health care that was delivered, such as differences among patients' expectation levels, can cause variability in satisfaction ratings, which reduce their reliability for widespread and ongoing monitoring of attempts to improve patient-centred care [15]. In contrast, experiences of care measures ask patients to indicate what actually happened during the process of care delivery, and so are less influenced by subjective patient expectations and provide more detailed information to health care providers and systems about where quality improvements are needed $[16,17]$. However, in order to accurately reflect the quality of care received and identify variations in patients' experiences, PROMs should meet recommended psychometric criteria for reliability (internal consistency, test re-test reliability), and validity (face, content, construct validity) [18-24].

There are few existing reviews that have assessed the psychometric properties of measures developed to identify patients' experiences of care across a range of settings and diseases [25-28]. Only one of these reviews evaluated the psychometric properties of quality of care measures designed specifically for cancer patients, but focused on satisfaction measures [27]. Further, this review [27] did not investigate the degree to which these quality of care measures assessed the six IOM-endorsed dimensions of patient-centred care [1].

This systematic review identified:

1) the degree to which PROMs developed to assess the quality of patient-centred cancer care since the publication of the IOM's "Crossing the Quality Chasm" report in 2001 have addressed the IOM's six endorsed dimensions of patient-centred care [1]; and

2) the psychometric properties of these measures.

\section{Methods}

\section{Search strategy and selection criteria}

The electronic databases Medline, PsycINFO, Current Contents, Embase, CINAHL and Scopus were searched to retrieve published studies outlining the development of PROMs designed to assess the quality of patient-centred cancer care. Given the IOM's Crossing the Quality Chasm report was published in 2001 [1], databases were searched between January 2001 and December 2011 inclusive. The following combinations of keywords were used: (patientcentred or patient-centered or quality of care or satisfaction or experience ${ }^{*}$ ) AND (questionnaire* or survey* or instrument* or measure ${ }^{*}$ or scale ${ }^{*}$ or tool*) AND (cancer* or neoplasm* or oncol*). The use of an * in the keywords allows words that contain that term to be captured in the literature search. For example the keyword measure* will identify articles that contain variations of that word such 
as measure, measures, measurement and measurements. The reference lists of retrieved articles were also checked to identify any additional relevant publications.

The inclusion criteria for this systematic review were studies that:

(i) reported the development and psychometric properties (reliability and validity) of new PROMs designed to assess the quality of patient-centred cancer care, or reported the validation of an existing measure for use with a new population (e.g. patient-centred care measure translated for use with a Spanish cancer patient population). Given the IOM's recommendations were published in 2001 [1], studies describing the validation of an existing measure were eligible only if the original PROM was developed from 2001 onwards.

(ii) described PROMs specifically developed for use with adult cancer patient populations (i.e., aged 18 years or older); and

(iii) were published in an English language peerreviewed journal.

Publications were excluded if they:

(i) were reviews, editorials, commentaries or protocol papers;

(ii) reported qualitative research or used a Delphi consensus process;

(iii) reported data from medical records, administrative databases or cancer registries (i.e., patients were not surveyed);

(iv) focussed on cancer screening only;

(v) predominately surveyed cancer patients under 18 years of age;

(vi) assessed the views of health professionals such as oncologists, nurses, and general practitioners;

(vii) examined the perceptions of relatives and/or caregivers;

(viii) included only cancer patients with advanced cancer or those receiving end of life care; These patients were excluded because the outcome measures and care delivered to patients with advanced cancer can be unique, reflecting the specific goals of advanced disease and/or end-oflife care [29].

(ix) reported only patient ratings of quality of care and/ or patient characteristics associated with quality of care - i.e. did not develop a measure with the aim of testing its psychometric properties; and

(x) validation of an existing measure that was not eligible for the review (e.g. the original PROM was developed prior to 2001). PROMs developed prior to 2001 were excluded because it would have been unreasonable to assess the degree to which such PROMs addressed the IOM's dimensions of patientcentred care given the IOM recommendations were published in 2001 [1].

\section{Study and sample characteristics}

The study and sample characteristics extracted from eligible publications included: the name of the measure; country of development; patient recruitment setting (e.g. hospital, cancer registry); patient eligibility criteria; sample size; consent rate; participants' socio-demographic characteristics (e.g. mean age, gender, level of education, employment status); and participants' disease and treatment characteristics (e.g. cancer type, cancer stage and/ or time since diagnosis, treatments received).

\section{Items and subscales of measures}

Information extracted about the characteristics of each measure included: the type of measure (i.e. satisfaction versus experiences); number of items; the type of response scale, and the names and number of subscales. Two coders (FT \& SKR) independently examined each of the PROMs' items to determine whether or not the PROM contained content that related to any of the IOM's six patient-centred dimensions and how many of the six IOM-endorsed dimensions of patient-centred care were covered [1]. At least one item in the PROM needed to examine issues related to a particular IOM patient-centred care dimension (as defined below) for that area to be categorised as addressed. A conservative approach was taken when deciding whether or not a measure covered a particular dimension. For example, if a measure included an item that examined whether a patient was provided with information on long-term side effects, the measure was categorised as meeting the information and communication dimension, but not the physical comfort dimension. The physical comfort dimension was classified as present only if items assessed the provision of pain relief or the management of physical symptoms. The criteria used to classify each patientcentred care dimension, which are based on the definitions outlined in the IOM's "Crossing the Quality Chasm" report [1], are described below. Only one aspect of the dimension was needed for the PROM to be classed as covering that patient-centred care dimension.

\section{1) Respect for patients' values, preferences, and expressed needs}

PROMs were classified as covering this dimension if they assessed: a) whether care responded to the patient's cultural and other values, preferences and needs; b) whether patients were given the opportunity to express their views; c) whether patients were treated with respect during care; and/or d) whether patients were 
informed and involved in decision making according to their preferences [1].

\section{2) Coordinated and integrated care}

PROMs were rated as containing this dimension if they asked: a) whether patient care was coordinated and integrated; b) whether there was timely transfer of up-todate patient information between healthcare professionals; and/or c) whether patient transitions from one healthcare setting to another went smoothly [1].

\section{3) Provide information, communication, and education}

PROMs met the criteria for this dimension if they examined whether health care professionals: a) communicated with patients in a way they could understand; and/or b) provided accurate information regarding care including diagnosis, prognosis, treatment options, follow-up care and support services, according to the patient's preferred level of information provision [1].

\section{4) Physical comfort}

PROMs were classified as covering this dimension if they asked patients whether health care professionals: a) promptly provided pain relief; and/or b) attended to the patient's physical symptoms and needs [1].

\section{5) Emotional support}

PROMs were categorised as meeting this dimension if they assessed whether healthcare professionals: a) addressed the patients' emotional and spiritual concerns, such as anxiety, which could be experienced for a variety of reasons including uncertainty about their disease, concerns about the financial impact of treatment, or worrying about the impact of the illness on their family [1].

\section{6) Involvement of family and friends}

PROMs were considered to have met this dimension if they assessed whether: a) family and friends were involved in the patient's decision making and care according to the patient's preferences; and/or b) whether care was responsive to the concerns of family and friends and recognised their needs [1].

Two coders (FT \& SKR) also independently examined which PROMs covered all aspects within each of the IOM dimensions. For instance in terms of the physical comfort dimension, PROMs that included items that addressed both of the following criteria were identified: a) promptly provided pain relief; and b) attended to the patient's physical symptoms and needs.

\section{Psychometric properties of measures}

The psychometric properties of each measure were assessed against the same criteria used by ClintonMcHarg and colleagues in their review of instruments designed to measure the psychosocial health of adolescent and young adult cancer survivors [30]. The psychometric criteria are described below.

\section{Internal consistency}

A measure was coded as having acceptable internal consistency if correlations for the total scale and each subscale were calculated [19] and a Cronbach's alpha $>0.70$ (continuous or dichotomous scales) or Kuder-Richardson 20 (KR-20) $>0.70$ was reported for the total scale and each sub-scale $[18,19]$.

\section{Test-retest reliability}

Measures were recorded as having adequate test-retest reliability if the instrument had been administered twice to the same sample and: 1) the second administration occurred within 2-14 days of the first administration [20]; and 2) correlations for the total scale, subscales and items were calculated [21] and the agreement between scores achieved a Cohen's kappa co-efficient $(\kappa)>0.60$ (nominal or ordinal scales) [19] or Pearson correlation coefficient $(r)>0.70$ (interval scales) $[18,19]$ or intraclass correlation coefficient (ICC) $>0.70$ (interval scales) $[18,19]$.

\section{Face validity}

Measures were considered to have face validity if both those who administered it, and those who completed it, agreed it appeared to measure what it was designed to measure [22].

\section{Content validity}

A measure was reported to have adequate content validity if the following processes were described: 1 ) how the items were developed or selected $[18,19]$; 2) how and by whom the content was assessed [18,19]; and 3) if modifications to the content were needed that the revisions addressed the issues identified $[18,19]$.

\section{Construct validity}

Each measure was assessed as having adequate construct validity if any of the following tests were performed: 1 ) comparison with other existing measures [19] resulting in Pearson correlation coefficients of $(r)>0.40$ (convergent validity) or $(r)<0.30$ (divergent validity) [23]; 2) comparison of scores on the measure differ significantly between groups with known differences (discriminative validity) [18]; or 3) factor analysis [19] with Eigenvalues set at $>1[24]$.

\section{Cross-cultural adaptation}

A measure was considered to have adequate crosscultural adaptation if a conceptually and linguistically equivalent version of the original form confirmed the 
reliability and validity reflected in the original measure [18].

\section{Coding process}

Two authors (FT \& SKR) independently assessed all potentially relevant publications to determine whether they met eligibility for inclusion in the review. There was $84 \%$ agreement between the two coders' ratings. Where discrepancies emerged, inconsistent ratings were discussed between the coders until consensus was reached. Both coders also independently extracted information for the Tables from included publications to ensure accuracy. The coders then compared the information extracted and discussed any inconsistencies until agreement was reached.

\section{Results}

\section{Study eligibility}

A total of 671 publications were identified from the electronic database searches and publication reference lists. Of these, 161 publications were reviews, editorials, commentaries or protocol papers, 40 reported qualitative research and 16 used a Delphi consensus process and were excluded. A further 108 papers reported data from medical records, administrative databases or cancer registries and 53 focussed on cancer screening only and were removed. Of the 293 remaining publications, 48 assessed the views of health professionals such as oncologists, nurses, and general practitioners, 44 focussed on the perceptions of relatives or caregivers, one related to cancer patients aged under 18 years, and 37 focused on an advanced cancer population and/or those receiving endof-life care and were excluded. Of the remaining 163 publications that surveyed adult cancer patients, 121 examined the prevalence of features of care and/or characteristics associated with patient experiences and 14 validated an existing measure that was not eligible for the review (e.g. the original PROM was developed prior to 2001). One paper that reported the development of the EORTC OUT-PATSAT35 was published in French and therefore excluded [31]. This left 27 papers that reported the development of an instrument and its psychometric properties with an adult cancer patient population, or reported the psychometric properties of a re-validated measure for use with a new population. In these papers, 21 unique PROMs were described (see Figure 1).

\section{Setting and Sample Characteristics}

Table 1 provides a detailed description of the setting and sample characteristics of the eligible studies [32-55]. Six studies were conducted in the USA [32,35,42-44,47], five in The Netherlands $[37,39,40,50,52]$, three in England $[41,49,54]$, two in France $[53,55]$, and one in Australia
[33], Canada [34], Europe and Asia [38], Germany [46] and Japan [48]. Seventeen studies recruited cancer patients from hospitals or treatment centres [33,34,38-44,46-50,53-55], whereas only one study recruited patients via a populationbased cancer registry [32]. The sample sizes in each study ranged from 82 to 2659 cancer patients and the consent rates varied from $43 \%$ to $85 \%$. Thirteen studies included more than one cancer type [32-35,38,41,43,44,46-48,52,55].

\section{Patient-centred care instruments}

The names of the PROMs included in the review are shown in Tables 1, 2, 3, 4 and 5. As shown in Table 2, 15 measures examined patients' experiences of care $[32,33,37,39-44,48-50,52,53,55]$ while 6 measured satisfaction $[34,35,38,46,47,54]$. The number of items for each measure ranged from 15 to 152 , and the number of subscales ranged from 1 to 15 . The type of response scales varied across the different instruments. The number of IOM-endorsed patient-centred care dimensions [1] that were included in each measure were as follows: two measures included one dimension [35,54], two measures had two dimensions $[42,46]$, seven measures had three dimensions $[34,39,41,47,48,50,55]$, five measures had four dimensions $[32,33,37,49,53]$, and four measures had five dimensions $[38,43,44,52]$. Only one measure, the Indicators (Non-small Cell Lung Cancer) measure, covered all six dimensions of patient-centred care [40]. Table 3 summarises the PROMs that addressed each of the IOM-endorsed patient-centred care dimensions.

Figure 2 illustrates the frequency with which the six IOM-endorsed patient-centred dimensions were included across the 21 measures. "Information, communication and education" was the dimension most commonly included (19 measures). In contrast, only five measures assessed the "involvement and wellbeing of family and friends". Thirteen measures addressed all the IOM criteria for the emotional support dimension [32-34,37-41,43,48,52,53,55], 8 measures for information, communication and education $[32,37,47,48,50,52,54,55]$ and one measure for physical comfort [44]. None of the measures addressed all the IOM criteria within the dimensions of respect for patient values, preferences and needs; coordinated and integrated care; and involvement and wellbeing of family and friends.

\section{Psychometric properties of instruments}

A description of the psychometric properties for each PROM is reported in Table 4.

\section{Internal consistency}

Seven of the 21 measures met the criteria considered adequate for internal consistency by reporting a Cronbach's alpha $>0.70$ for both the total scale and each sub-scale $[33,42,43,46-48,55]$. Of the 13 studies that reported 


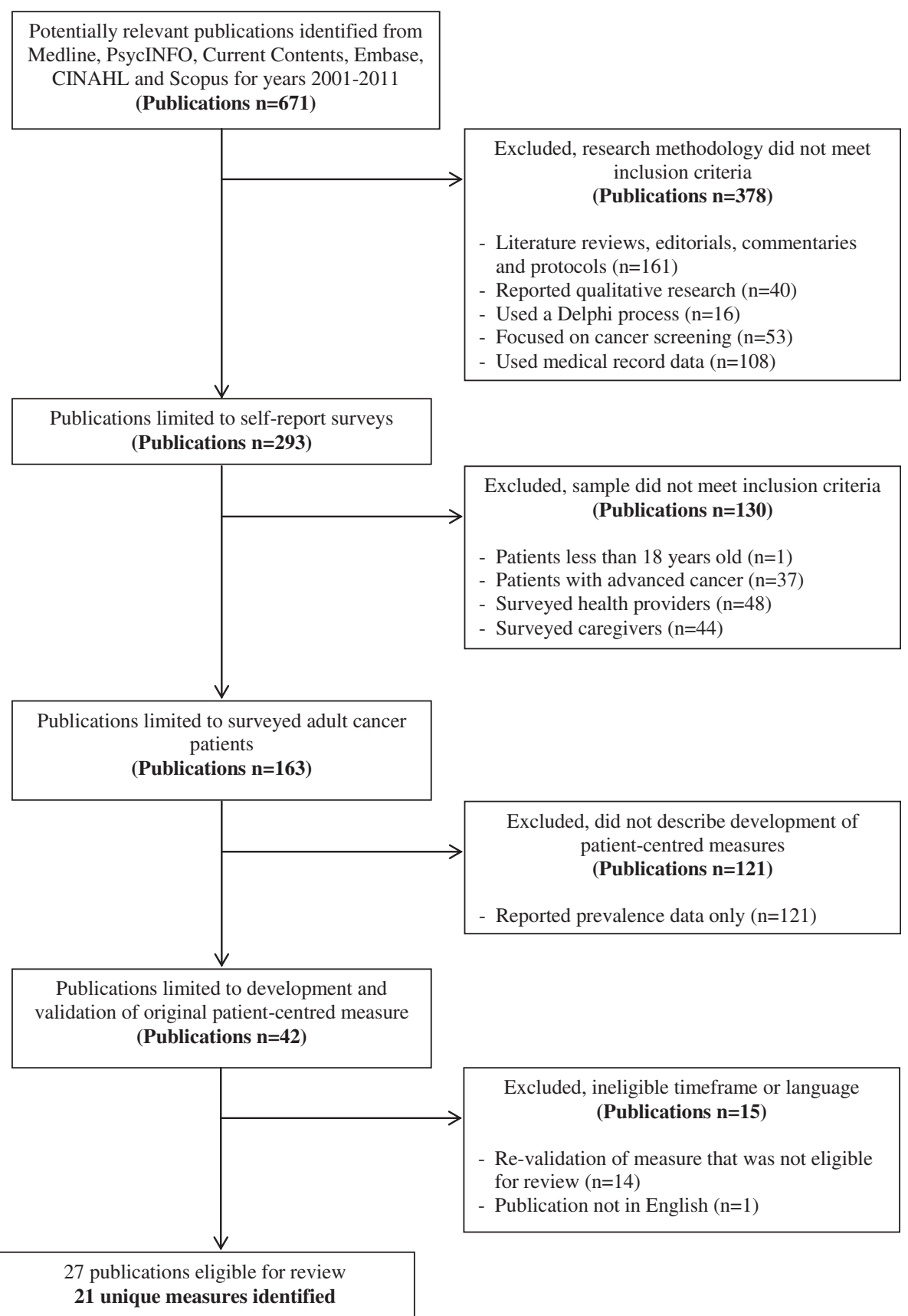

Figure 1 Flowchart of methods used to identify relevant publications.

Cronbach's alpha only for the PROMs' subscales, six of these measures showed all subscales had a Cronbach's alpha $>0.70[34,35,44,52-54]$.

\section{Test-retest reliability}

None of the five measures that examined test-retest reliability $[33,35,38,49,53]$ met recommended adequacy criteria of a second administration within 2-14 days of the first administration [20] and an adequate agreement between the two administrations on scores for the total scale, subscales and items $[18,19]$.

\section{Face/content validity}

Fifteen measures met the criteria considered adequate for face validity and content validity [33,35,37-41, $43,44,48-50,52,53,55]$. 
Table 1 Sample characteristics of studies that have developed PROMs assessing quality of patient-centred cancer care

\begin{tabular}{|c|c|c|c|c|c|c|c|}
\hline Measure & $\begin{array}{l}\text { Sample } \\
\text { size }\end{array}$ & Consent rate & Eligibility criteria & Setting \& country & Socio-demographics & $\begin{array}{l}\text { Cancer type, } \\
\text { stage/ diagnosis }\end{array}$ & Cancer treatments \\
\hline \multirow{6}{*}{$\begin{array}{l}\text { Assessment of Patient } \\
\text { Experiences of Cancer } \\
\text { Care (APECC) [32] }\end{array}$} & \multirow[t]{6}{*}{623} & $\begin{array}{l}69.2 \% \text { participation } \\
\text { rate. }\end{array}$ & \multirow{6}{*}{$\begin{array}{l}\text { Read English, diagnosed with leukaemia } \\
\text { or bladder or colorectal cancer between } \\
\text { June } 1999-\text { May } 2001 \text { (i.e., 2-5 years before } \\
\text { study enrolment), at least } 20 \text { years old at } \\
\text { diagnosis, have received cancer treatment, } \\
\text { have the cancer of interest as their first } \\
\text { cancer diagnosis, not have any other cancer } \\
\text { between their initial diagnosis and the start } \\
\text { of the study, have no objections from their } \\
\text { physician of record to their participation. }\end{array}$} & \multirow{6}{*}{$\begin{array}{l}\text { Cancer Prevention } \\
\text { Institute of California's } \\
\text { cancer registry, USA. }\end{array}$} & $43.3 \%$ women & Colorectal cancer: 59.6\% & $38.7 \%$ surgery only \\
\hline & & \multirow[t]{5}{*}{$\begin{array}{l}49.2 \% \text { response } \\
\text { rate. }\end{array}$} & & & $\begin{array}{l}37.7 \% \text { aged } \\
50-64 \text { years }\end{array}$ & Bladder cancer: 26.2\% & \multirow[t]{3}{*}{$\begin{array}{l}35.1 \% \text { surgery plus } \\
\text { chemotherapy or } \\
\text { radiation }\end{array}$} \\
\hline & & & & & $\begin{array}{l}20.3 \% \text { college } \\
\text { degree }\end{array}$ & Leukaemia: 14.3\% & \\
\hline & & & & & $\begin{array}{l}71.5 \% \text { married/ } \\
\text { defacto }\end{array}$ & & \\
\hline & & & & & $\begin{array}{l}\text { 73.8\% Non- } \\
\text { Hispanic white }\end{array}$ & $84.4 \%$ in remission. & $\begin{array}{l}12.3 \% \text { surgery plus } \\
\text { chemotherapy and } \\
\text { radiation }\end{array}$ \\
\hline & & & & & $\begin{array}{l}83.6 \% \text { private } \\
\text { health insurance }\end{array}$ & $\begin{array}{l}\text { Mean of } 3.5 \text { years since } \\
\text { diagnosis. }\end{array}$ & $\begin{array}{l}13.9 \% \text { chemotherapy } \\
\text { with or without } \\
\text { radiation but no } \\
\text { surgery }\end{array}$ \\
\hline \multirow{8}{*}{$\begin{array}{l}\text { Cancer Care Coordination } \\
\text { Questionnaire for } \\
\text { Patients [33] }\end{array}$} & \multirow[t]{8}{*}{686} & \multirow[t]{8}{*}{-} & \multirow{7}{*}{$\begin{array}{l}\text { Sample } 1(n=245) \text { : Patients were in follow-up } \\
\text { for any cancer that had been treated between } \\
3 \text { to } 12 \text { months previously, had sufficient } \\
\text { English and were not cognitively impaired } \\
\text { and were not receiving end of life care. }\end{array}$} & \multirow{4}{*}{$\begin{array}{l}\text { Sample 1: Six centres } \\
\text { ( } 2 \text { metropolitan } \\
\text { \& } 4 \text { regional). }\end{array}$} & Total participants: & Colorectal: 82.5\% & $96 \%$ surgery \\
\hline & & & & & $46.8 \%$ women & Gynaecological: 7.6\% & 40.5\% chemotherapy \\
\hline & & & & & Mean age: 66.1 years & Breast: $2.6 \%$ & $12.2 \%$ radiotherapy \\
\hline & & & & & $66.9 \%$ married/defacto & $\begin{array}{l}\text { Lung/mesothelioma: } \\
1.3 \%\end{array}$ & \multirow[t]{5}{*}{$\begin{array}{l}3.9 \% \text { hormone } \\
\text { therapy }\end{array}$} \\
\hline & & & & \multirow{3}{*}{$\begin{array}{l}\text { Sample 2: } 22 \text { public } \\
\text { and private hospitals } \\
\text { in metropolitan and } \\
\text { regional centres. }\end{array}$} & $\begin{array}{l}35.3 \% \text { tertiary degree } \\
\text { or diploma }\end{array}$ & $\begin{array}{l}\text { Other/multiple sites: } \\
4.7 \%\end{array}$ & \\
\hline & & & & & $\begin{array}{l}23.5 \% \text { employed } \\
\text { full-time }\end{array}$ & Primary cancer: $91.8 \%$ & \\
\hline & & & & & & Recurrent cancer: 3.8\% & \\
\hline & & & $\begin{array}{l}\text { Sample } 2(n=441) \text { : Patients with a newly } \\
\text { diagnosed colorectal cancer undergoing } \\
\text { initial surgical treatment. }\end{array}$ & Australia & & & \\
\hline \multirow{9}{*}{$\begin{array}{l}\text { Cancer Patient Information } \\
\text { Importance and Satisfaction } \\
\text { Tool [34] }\end{array}$} & \multirow[t]{9}{*}{540} & \multirow[t]{9}{*}{-} & \multirow[t]{9}{*}{-} & \multirow{9}{*}{$\begin{array}{l}\text { Ambulatory setting of } \\
\text { regional cancer centre, } \\
\text { Canada. }\end{array}$} & $53 \%$ women & Breast: $19.1 \%$ & \multirow[t]{9}{*}{-} \\
\hline & & & & & Mean age: 60.9 years & Haematological: 12.5\% & \\
\hline & & & & & & Genitourinary: $12.5 \%$ & \\
\hline & & & & & & Skin: $11.5 \%$ & \\
\hline & & & & & & Gastrointestinal: 11.5\% & \\
\hline & & & & & & Head and neck: $11.3 \%$ & \\
\hline & & & & & & Gynecologic: 11.2\% & \\
\hline & & & & & & Lung: $10.4 \%$ & \\
\hline & & & & & & $\begin{array}{l}21.3 \% \text { diagnosed in last } \\
\text { year, } 48.3 \% \text { between } \\
2-5 \text { years ago. }\end{array}$ & \\
\hline
\end{tabular}

列 previously, had sufficient (2 metropolitan Questionnaire for .

Cancer Patient Information and Satisfaction Tool [34] 
Table 1 Sample characteristics of studies that have developed PROMs assessing quality of patient-centred cancer care (Continued)

\begin{tabular}{|c|c|c|c|c|c|c|c|}
\hline \multirow[t]{7}{*}{$\begin{array}{l}\text { Cancer Therapy Satisfaction } \\
\text { Questionnaire (CTSQ) [35,36] }\end{array}$} & \multirow[t]{7}{*}{361} & \multirow[t]{7}{*}{-} & \multirow{7}{*}{$\begin{array}{l}\text { Provided written informed consent, aged } \\
18 \text { years or older, read and write in English, } \\
\text { available for follow-up evaluation, actively } \\
\text { receiving more than one cycle of first- or } \\
\text { second-line chemo, biological or hormonal } \\
\text { therapy for early or advanced cancer, } \\
\text { mentally and physically capable of } \\
\text { participation. }\end{array}$} & \multirow[t]{7}{*}{$\begin{array}{l}14 \text { community clinical } \\
\text { practices, USA. }\end{array}$} & $\begin{array}{l}63.2 \% \text { women } \\
\text { Mean age: } 60.7 \text { years }\end{array}$ & Colorectal: 33.5\% & $\begin{array}{l}\text { First-line of therapy: } \\
48.8 \%\end{array}$ \\
\hline & & & & & $90.9 \%$ white & Lung: $25.2 \%$ & \multirow[t]{2}{*}{$\begin{array}{l}\text { Second-line of } \\
\text { therapy: } 28.0 \%\end{array}$} \\
\hline & & & & & \multirow{5}{*}{$\begin{array}{l}16.3 \% \text { college/university } \\
\text { degree }\end{array}$} & Melanoma: 3.3\% & \\
\hline & & & & & & Stage l: 6.1\% & \multirow[t]{4}{*}{ Adjuvant: 23.3\% } \\
\hline & & & & & & Stage II: $25.2 \%$ & \\
\hline & & & & & & Stage III: $24.9 \%$ & \\
\hline & & & & & & Stage IV: $43.8 \%$ & \\
\hline \multirow{4}{*}{$\begin{array}{l}\text { Consumer Quality Index } \\
\text { Breast Care (CQI-BC) [37] }\end{array}$} & \multirow[t]{4}{*}{731} & \multirow[t]{4}{*}{$63 \%$} & \multirow{4}{*}{$\begin{array}{l}\text { Older than } 18 \text { years, having received breast } \\
\text { care in the last } 24 \text { months, not being } \\
\text { approached in the past for CQI surveys. }\end{array}$} & \multirow{4}{*}{$\begin{array}{l}\text { Selected from claims } \\
\text { data of four health } \\
\text { insurance companies, } \\
\text { The Netherlands. }\end{array}$} & 99.7\% women & Breast cancer: $57 \%$ & \multirow[t]{4}{*}{-} \\
\hline & & & & & $\begin{array}{l}30 \% \text { aged between } \\
55 \text { and } 64 \text { years }\end{array}$ & $\begin{array}{l}\text { Benign breast disorder: } \\
38 \%\end{array}$ & \\
\hline & & & & & & $\begin{array}{l}\text { Breast carcinoma in situ: } \\
5 \%\end{array}$ & \\
\hline & & & & & & - & \\
\hline \multirow{14}{*}{$\begin{array}{l}\text { EORTC cancer in-patient } \\
\text { satisfaction with care } \\
\text { measure (EORTC } \\
\text { IN-PATSAT32) [38] }\end{array}$} & \multirow[t]{14}{*}{647} & \multirow[t]{14}{*}{$84.9 \%$} & \multirow{14}{*}{$\begin{array}{l}\text { Diagnosed with cancer, aged } 18 \text { years or } \\
\text { older, hospitalized for at least three days, } \\
\text { mentally able to complete questionnaire. }\end{array}$} & \multirow{14}{*}{$\begin{array}{l}\text { Surgery or medical } \\
\text { oncology wards in } \\
\text { hospitals, Belgium, } \\
\text { France, Germany, } \\
\text { Italy, Poland, Spain, } \\
\text { Sweden, Taiwan, } \\
\text { United Kingdom. }\end{array}$} & $59 \%$ women & Breast: $35.1 \%$ & $\begin{array}{l}\text { Current or planned } \\
\text { treatment: }\end{array}$ \\
\hline & & & & & Median age: 57 years & Gastro-intestinal: 17.2\% & $55 \%$ surgery \\
\hline & & & & & $21 \%$ university educated & Gynaecologic: 10\% & \\
\hline & & & & & 73.6\% married/defacto & Head and neck: $7.3 \%$ & 40.8\% chemotherapy \\
\hline & & & & & $40.5 \%$ full time employed & Genito-urinary: $7.1 \%$ & $\begin{array}{l}3.2 \% \text { surgery and } \\
\text { chemotherapy }\end{array}$ \\
\hline & & & & & & Haematological: $6.5 \%$ & \\
\hline & & & & & & Respiratory: 5.6\% & $0.9 \%$ other \\
\hline & & & & & & Bone: $2.8 \%$ & \\
\hline & & & & & & Brain: 1.5\% & \\
\hline & & & & & & Melanoma: 0.8\% & \\
\hline & & & & & & Other: $6.2 \%$ & \\
\hline & & & & & & $\begin{array}{l}73.9 \% \text { local/loco- } \\
\text { regional }\end{array}$ & \\
\hline & & & & & & $26 \%$ metastatic & \\
\hline & & & & & & $\begin{array}{l}\text { Median of } 15 \text { weeks } \\
\text { since diagnosis. }\end{array}$ & \\
\hline \multirow{3}{*}{$\begin{array}{l}\text { Indicators (Head \& Neck } \\
\text { Cancer) [39] }\end{array}$} & \multirow[t]{3}{*}{158} & \multirow[t]{3}{*}{$84 \%$} & \multirow{3}{*}{$\begin{array}{l}\text { Patients with head and neck cancer newly } \\
\text { diagnosed between May to December } 2003\end{array}$} & \multirow{3}{*}{$\begin{array}{l}\text { Selected from clinic } \\
\text { lists at a university } \\
\text { hospital, The } \\
\text { Netherlands }\end{array}$} & $27 \%$ women & Head \& neck cancer. & First treatment: \\
\hline & & & & & Mean age: 62 years & $\begin{array}{l}\text { Larynx and hypharynx: } \\
38 \%\end{array}$ & Operation: 56\% \\
\hline & & & & & $23 \%$ highly educated & & Radiotherapy: 37\% \\
\hline
\end{tabular}


Table 1 Sample characteristics of studies that have developed PROMs assessing quality of patient-centred cancer care (Continued)

\begin{tabular}{|c|c|c|c|c|c|c|c|}
\hline & & & & & & $\begin{array}{l}\text { Cavity of the mouth: } \\
36 \%\end{array}$ & \\
\hline & & & & & & Other: $26 \%$ & Chemotherapy: 7\% \\
\hline & & & & & & - & \\
\hline \multirow[t]{2}{*}{$\begin{array}{l}\text { Indicators (Non-small } \\
\text { Cell Lung Cancer) [40] }\end{array}$} & \multirow[t]{2}{*}{100} & \multirow[t]{2}{*}{$76 \%$} & \multirow{2}{*}{$\begin{array}{l}\text { Patients newly diagnosed with non-small } \\
\text { cell lung cancer between September } 2004 \\
\text { and February } 2005 .\end{array}$} & \multirow[t]{2}{*}{$\begin{array}{l}6 \text { hospitals, The } \\
\text { Netherlands. }\end{array}$} & $34 \%$ women & $\begin{array}{l}\text { Non-small cell lung } \\
\text { cancer. }\end{array}$ & \multirow[t]{2}{*}{-} \\
\hline & & & & & Mean age: 66 years & Stage IV: $24 \%$ & \\
\hline \multirow{17}{*}{$\begin{array}{l}\text { Medical Care Questionnaire } \\
\text { (MCQ) [41] }\end{array}$} & Phase & Phase 3: & \multirow{17}{*}{$\begin{array}{l}\text { Adult patients from all tumor groups attending } \\
\text { the Medical Oncology Unit, could read and } \\
\text { understand English, were not exhibiting overt } \\
\text { cognitive dysfunction or signs of distress. }\end{array}$} & \multirow{17}{*}{$\begin{array}{l}\text { A regional hospital, } \\
\text { England. }\end{array}$} & Phase 3: & Phase 3: & \multirow[t]{17}{*}{-} \\
\hline & $3: 200$ & $70 \%$ & & & $81 \%$ women & Gynecological: 38\% & \\
\hline & \multirow[t]{15}{*}{ Phase 4: 477} & \multirow[t]{15}{*}{ Phase 4: 79.6\% } & & & $42 \%$ aged $45-59$ years & Breast: $26.5 \%$ & \\
\hline & & & & & $74 \%$ married/de facto & Genitourinary: $16.5 \%$ & \\
\hline & & & & & 13\% employed full time & Sarcoma: $5.5 \%$ & \\
\hline & & & & & & Gastrointestinal: $4.5 \%$ & \\
\hline & & & & & Phase 4: & Melanoma: $1 \%$ & \\
\hline & & & & & $74.2 \%$ women & Other: 8\% & \\
\hline & & & & & $40.9 \%$ aged $45-59$ years & & \\
\hline & & & & & $75.1 \%$ married/de facto & Phase 4: & \\
\hline & & & & & 44.2\% employed full time & Gynecological: 33.8\% & \\
\hline & & & & & & Breast: $23.5 \%$ & \\
\hline & & & & & & Genitourinary: $21.4 \%$ & \\
\hline & & & & & & Melanoma: 8.4\% & \\
\hline & & & & & & Sarcoma: $7.5 \%$ & \\
\hline & & & & & & Other: $5.5 \%$ & \\
\hline & & & & & & - & \\
\hline \multirow{6}{*}{$\begin{array}{l}\text { Modified Version of the } \\
\text { Perceived Involvement in } \\
\text { Care Scale (M-PICS) [42] }\end{array}$} & \multirow[t]{6}{*}{87} & \multirow[t]{6}{*}{$74 \%$} & \multirow{6}{*}{$\begin{array}{l}\text { Females aged } 18 \text { years or older, confirmed } \\
\text { diagnosis of breast cancer, reported pain of } \\
\text { at least moderate intensity (score } \geq 4 \text { on the } \\
\text { Brief Pain Inventony's Worst Pain Intensity } \\
\text { item) over prior two weeks, absence of any } \\
\text { gross cognitive impairment, literate in English } \\
\text { or Spanish. }\end{array}$} & \multirow{6}{*}{$\begin{array}{l}\text { Four hospital-based } \\
\text { outpatient oncology } \\
\text { clinics and a private } \\
\text { hospital-affliated } \\
\text { oncology practice, } \\
\text { USA. }\end{array}$} & $100 \%$ women & Breast cancer. & 89.7\% chemotherapy \\
\hline & & & & & Mean age: 50.4 years & Stage l: $12.6 \%$ & \multirow{5}{*}{$\begin{array}{l}43.7 \% \text { radiation } \\
\text { therapy }\end{array}$} \\
\hline & & & & & $31 \%$ Caucasian & Stage II: $24.1 \%$ & \\
\hline & & & & & $50.6 \%$ married/partnered & Stage III: 13.8\% & \\
\hline & & & & & $\begin{array}{l}64.5 \% \text { at least } 13 \text { years } \\
\text { education }\end{array}$ & Stage IV: $49.4 \%$ & \\
\hline & & & & & $26.2 \%$ employed & & \\
\hline \multirow{5}{*}{$\begin{array}{l}\text { Oncology Patients' } \\
\text { Perceptions of the } \\
\text { Quality of Nursing Care } \\
\text { Scale (OPPQNCS) [43] }\end{array}$} & \multirow[t]{5}{*}{436} & \multirow[t]{5}{*}{-} & \multirow{5}{*}{$\begin{array}{l}18 \text { years or older, registered with the receptionist } \\
\text { on the days of data collection, had received } \\
\text { cancer nursing care in the clinic or hospital, not } \\
\text { extremely ill or confused. }\end{array}$} & \multirow{5}{*}{$\begin{array}{l}\text { A haematology- } \\
\text { oncology clinic, } \\
\text { USA. }\end{array}$} & $66 \%$ women & Breast: 40\% & \multirow[t]{5}{*}{ In active treatment } \\
\hline & & & & & Mean age: 54.8 years & Melanoma: 9\% & \\
\hline & & & & & 93\% white & Lung: 6\% & \\
\hline & & & & & \multirow{2}{*}{$\begin{array}{l}81 \% \text { more than high } \\
\text { school education }\end{array}$} & Renal cell: $4 \%$ & \\
\hline & & & & & & Squamous cell: $4 \%$ & \\
\hline
\end{tabular}




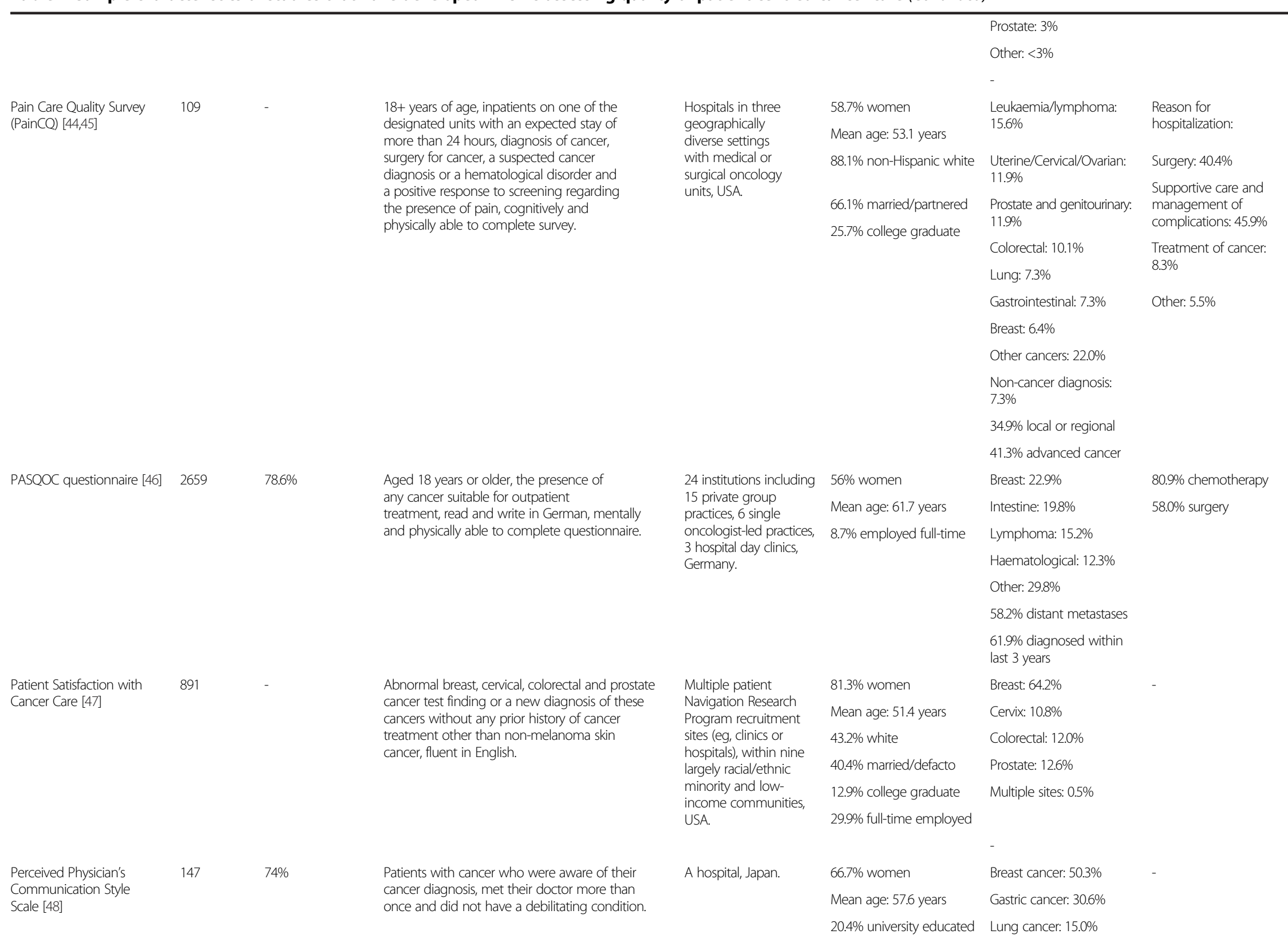


Table 1 Sample characteristics of studies that have developed PROMs assessing quality of patient-centred cancer care (Continued)

\begin{tabular}{|c|c|c|c|c|c|c|c|}
\hline & & & & & & Other cancer: $4.1 \%$ & \\
\hline & & & & & & $55.1 \%$ disease free & \\
\hline & & & & & & $\begin{array}{l}44.9 \% \text { recurrent or } \\
\text { metastatic disease }\end{array}$ & \\
\hline \multirow{4}{*}{$\begin{array}{l}\text { Prostate Care Questionnaire } \\
\text { for Patients (PCQ-P) [49] }\end{array}$} & \multirow[t]{4}{*}{865} & \multirow[t]{4}{*}{$69.2 \%$} & \multirow{4}{*}{$\begin{array}{l}\text { Patients diagnosed with, or treated for prostate } \\
\text { cancer within the past two years, who were } \\
\text { not too ill to participate. }\end{array}$} & \multirow[t]{4}{*}{5 hospitals, England } & $100 \%$ male & \multirow[t]{2}{*}{ Prostate cancer } & \multirow[t]{4}{*}{-} \\
\hline & & & & & $40.5 \%$ aged $65-74$ years & & \\
\hline & & & & & $92.8 \%$ white & - & \\
\hline & & & & & 21.3\% employed & & \\
\hline \multirow{4}{*}{$\begin{array}{l}\text { QUOTE Breast } \\
\text { Cancer [50,51] }\end{array}$} & \multirow[t]{4}{*}{276} & \multirow[t]{4}{*}{$43 \%$} & \multirow{4}{*}{$\begin{array}{l}\text { Experience with any type of surgery for breast } \\
\text { cancer } 3-15 \text { months before the start of the study, } \\
\text { age older than } 17 \text { years and mental competence } \\
\text { as judged by the breast nurse. }\end{array}$} & \multirow{4}{*}{$\begin{array}{l}5 \text { hospitals, The } \\
\text { Netherlands. }\end{array}$} & $100 \%$ women & \multirow[t]{2}{*}{ Breast cancer } & $54 \%$ lumpectomy \\
\hline & & & & & Mean age: 57 years & & $\begin{array}{l}52 \% \text { (modified) } \\
\text { radical mastectomy }\end{array}$ \\
\hline & & & & & \multirow{2}{*}{$\begin{array}{l}32 \% \text { college/university } \\
\text { educated }\end{array}$} & \multirow{2}{*}{$\begin{array}{l}16 \% \text { diagnosed } 3-6 \text { months } \\
\text { ago, } 47 \% 7-12 \text { months } \\
\text { ago, 34\% 13-18 months } \\
\text { ago, 3\% more than } \\
18 \text { months ago. }\end{array}$} & $50 \%$ radiotherapy \\
\hline & & & & & & & $38 \%$ chemotherapy \\
\hline \multirow[t]{9}{*}{ QUOTE ${ }^{\text {chemo }}[52]$} & \multirow[t]{9}{*}{345} & \multirow[t]{9}{*}{$59.3 \%$} & \multirow{9}{*}{$\begin{array}{l}60 \text { most recent patients from each hospital who } \\
\text { were new to chemotherapy, aged } 18 \text { years or } \\
\text { older, able to read Dutch. }\end{array}$} & \multirow{9}{*}{$\begin{array}{l}\text { Hospital records } \\
\text { from } 10 \text { hospitals, } \\
\text { The Netherlands. }\end{array}$} & $67 \%$ women & Breast: $47.2 \%$ & \multirow{2}{*}{$\begin{array}{l}16.2 \% \text { chemotherapy } \\
\text { only }\end{array}$} \\
\hline & & & & & Mean age: 55.7 years & \multirow{2}{*}{$\begin{array}{l}\text { Digestive-gastrointestinal: } \\
21.5 \%\end{array}$} & \\
\hline & & & & & 79\% lived with partner & & \multirow{2}{*}{$\begin{array}{l}74.5 \% \text { chemotherapy } \\
\& \text { surgery }\end{array}$} \\
\hline & & & & & $28 \%$ highly educated & $\begin{array}{l}\text { Haematologic: } 10.6 \% \\
\text { Lung: } 9.7 \%\end{array}$ & \\
\hline & & & & & \multirow[t]{5}{*}{$52.8 \%$ employed } & $\begin{array}{l}\text { Gynaecological: } 6.2 \% \\
\text { Urologic: } 2.9 \%\end{array}$ & $\begin{array}{l}45.5 \% \text { chemotherapy } \\
\text { \& radiotherapy }\end{array}$ \\
\hline & & & & & & Other: $1.8 \%$ & $\begin{array}{l}21.1 \% \text { chemotherapy } \\
\text { \& hormone replacement } \\
\text { therapy }\end{array}$ \\
\hline & & & & & & $\begin{array}{l}\text { Mean of } 11.5 \text { months } \\
\text { since diagnosis. }\end{array}$ & $\begin{array}{l}6.2 \% \text { chemotherapy } \\
\& \text { immunotherapy }\end{array}$ \\
\hline & & & & & & & $74.8 \%$ curative intent \\
\hline & & & & & & & $25.2 \%$ palliative intent \\
\hline \multirow[t]{4}{*}{ REPERES-60 [53] } & \multirow[t]{4}{*}{820} & \multirow[t]{4}{*}{$84 \%$} & \multirow{4}{*}{$\begin{array}{l}\text { A first diagnosis of invasive non-metastatic breast } \\
\text { cancer, at least two contacts for cancer with one } \\
\text { of the health professionals in one of the two } \\
\text { regions between diagnosis and the first year of } \\
\text { follow-up, signed informed consent. }\end{array}$} & \multirow{4}{*}{$\begin{array}{l}\text { Public and private } \\
\text { cancer centres in } \\
\text { two regions, France. }\end{array}$} & 100\% women & \multirow[t]{4}{*}{ Breast cancer. } & \multirow[t]{4}{*}{-} \\
\hline & & & & & Mean age: 58 years & & \\
\hline & & & & & $\begin{array}{l}55.1 \% \text { living with } \\
\text { spouse/partner }\end{array}$ & & \\
\hline & & & & & $\begin{array}{l}15.7 \% \text { higher education } \\
43.7 \% \text { employed }\end{array}$ & & \\
\hline \multirow{3}{*}{$\begin{array}{l}\text { Satisfaction with Cancer } \\
\text { Information Profile } \\
\text { (SCIP) [54] }\end{array}$} & 82 & $76 \%$ & Newly diagnosed patients with head and neck & 4 hospitals, England. & $34 \%$ women & Head and neck cancer. & Planned treatments: \\
\hline & & & & & & $\begin{array}{l}\text { Most common sites } \\
\text { tongue and laryngeal/ }\end{array}$ & $27 \%$ surgery only \\
\hline & & & & & & & $\begin{array}{l}26 \% \text { radiotherapy } \\
\text { only }\end{array}$ \\
\hline
\end{tabular}

60 most recent patients from each hospital who Hospital records older, able to read Dutch.

A first diagnosis of invasive non-metastatic breast
cancer, at least two contacts for cancer with one of the health professionals in one of the two regions between diagnosis and the first year of follow-up, signed informed consent.

glottis 
Table 1 Sample characteristics of studies that have developed PROMs assessing quality of patient-centred cancer care (Continued)

\begin{tabular}{|c|c|c|c|c|c|c|c|}
\hline & & & & & & & $\begin{array}{l}31 \% \text { surgery and } \\
\text { radiotherapy }\end{array}$ \\
\hline & & & & & Mean age: 60 years & $\begin{array}{l}\text { Stages I and II: } \\
\text { approximately 50\%; }\end{array}$ & $\begin{array}{l}11 \% \text { radiotherapy } \\
\text { and chemotherapy }\end{array}$ \\
\hline & & & & & $\begin{array}{l}92 \% \text { white } \\
61 \% \text { married/de facto }\end{array}$ & $\begin{array}{l}\text { Stages III and IV: } \\
\text { approximately 50\% }\end{array}$ & $\begin{array}{l}5 \% \text { surgery, } \\
\text { radiotherapy } \\
\text { and chemotherapy }\end{array}$ \\
\hline \multirow[t]{7}{*}{ SAT-RAR [55] } & \multirow[t]{7}{*}{297} & \multirow[t]{7}{*}{$55.8 \%$} & \multirow{7}{*}{\multicolumn{2}{|c|}{$\begin{array}{l}\text { Aged }>18 \text { years, curative irradiation and satisfactory } 16 \text { centers, France. } \\
\text { general status ( }<3 \text { on WHO performance scale). }\end{array}$}} & Breast cancer $(n=98)$ : & Non-small cell lung & 100\% radiotherapy \\
\hline & & & & & Mean age: 56 years & cancer. & \multirow{3}{*}{$\begin{array}{l}\text { Respiratory gating: } \\
44 \% \text { (breast cancer) } \\
\& 67 \% \text { (lung cancer) }\end{array}$} \\
\hline & & & & & $100 \%$ women & Breast cancer. & \\
\hline & & & & & $\begin{array}{l}\text { Mean days hospitalized: } \\
4.7 \text { days }\end{array}$ & - & \\
\hline & & & & & $\begin{array}{l}\text { Non-small cell lung } \\
\text { cancer }(n=199) \text { : }\end{array}$ & & \multirow{3}{*}{$\begin{array}{l}1 \text { or more acute } \\
\text { toxicity during } \\
\text { treatment: } 96 \% \\
\text { (breast cancer) \& } \\
86 \% \text { (lung cancer) }\end{array}$} \\
\hline & & & & & $\begin{array}{l}\text { Mean age: } 65 \text { years } \\
15 \% \text { women }\end{array}$ & & \\
\hline & & & & & $\begin{array}{l}\text { Mean days hospitalized: } \\
3.8 \text { days Mean number } \\
\text { of hospitalizations: } 7.8\end{array}$ & & \\
\hline
\end{tabular}


Table 2 Measurement features of PROMs and included IOM-endorsed patient-centred care dimensions

\begin{tabular}{|c|c|c|c|c|c|}
\hline Measure & $\begin{array}{l}\text { Satisfaction/ } \\
\text { experience }\end{array}$ & Number of items & Response scale & Subscales & IOM patient-centred dimensions \\
\hline \multirow{14}{*}{$\begin{array}{l}\text { Assessment of Patient } \\
\text { Experiences of Cancer } \\
\text { Care (APECC) [32] }\end{array}$} & \multirow[t]{14}{*}{ Experiences } & \multirow[t]{14}{*}{33} & \multirow{2}{*}{$\begin{array}{l}\text { Not a problem, A small problem, } \\
\text { A big problem. }\end{array}$} & Getting needed care & Emotional support \\
\hline & & & & Timeliness of care & Information \& communication \\
\hline & & & \multirow{4}{*}{$\begin{array}{l}\text { Never, Sometimes, Usually, } \\
\text { Always. }\end{array}$} & Waiting time in physician's office & Integrated \& coordinated care \\
\hline & & & & Information exchange & Respectful to patients' values \\
\hline & & & & Physicians' affective behavior & \\
\hline & & & & Physicians' knowledge & \\
\hline & & & \multirow{4}{*}{$\begin{array}{l}\text { On time, }<15 \text { minutes, } 16-30 \text { minutes, } \\
31-45 \text { minutes, }>45 \text { minutes. }\end{array}$} & Interaction with nurses & \\
\hline & & & & Interaction with office staff & \\
\hline & & & & Health promotion & \\
\hline & & & & Coordination of care & \\
\hline & & & Poor, Fair, Good, Very good, Excellent. & Overall rating of care & \\
\hline & & & Yes definitely, Yes somewhat, No. & & \\
\hline & & & $\begin{array}{l}0 \text { (worst doctor possible) to } 10 \text { (best } \\
\text { doctor possible) }\end{array}$ & & \\
\hline & & & $\begin{array}{l}\text { Definitely yes, Probably yes, Not sure, } \\
\text { Probably not, Definitely not. }\end{array}$ & & \\
\hline \multirow{4}{*}{$\begin{array}{l}\text { Cancer Care Coordination } \\
\text { Questionnaire for Patients } \\
\text { [33] }\end{array}$} & \multirow[t]{4}{*}{ Experiences } & \multirow[t]{4}{*}{20} & \multirow{2}{*}{$\begin{array}{l}\text { Strongly disagree, Disagree, Neutral, } \\
\text { Agree, Strongly agree. }\end{array}$} & Communication & Emotional support \\
\hline & & & & Navigation & Family \& friends \\
\hline & & & \multirow{2}{*}{$\begin{array}{l}\text { Never, Rarely, Sometimes, Frequently, } \\
\text { Always. }\end{array}$} & & Information \& communication \\
\hline & & & & & Integrated \& coordinated care \\
\hline \multirow{3}{*}{$\begin{array}{l}\text { Cancer Patient Information } \\
\text { Importance and Satisfaction } \\
\text { Tool [34] }\end{array}$} & \multirow[t]{3}{*}{ Satisfaction } & \multirow[t]{3}{*}{24} & \multirow{2}{*}{$\begin{array}{l}5 \text { point scale from Not important (0) to } \\
\text { Very important (4). }\end{array}$} & Information importance & Emotional support \\
\hline & & & & Information satisfaction & Information \& communication \\
\hline & & & $\begin{array}{l}5 \text { point scale from Not satisfied (0) to } \\
\text { Very satisfied (4). }\end{array}$ & & Physical comfort \\
\hline \multirow{3}{*}{$\begin{array}{l}\text { Cancer Therapy Satisfaction } \\
\text { Questionnaire (CTSQ) }[35,36]\end{array}$} & \multirow[t]{3}{*}{ Satisfaction } & \multirow[t]{3}{*}{16} & \multirow{3}{*}{$\begin{array}{l}5 \text { point scale with } 1 \text { representing the } \\
\text { worst response and } 5 \text { representing } \\
\text { the best response. }\end{array}$} & Expectation of therapy & Respectful to patients' values \\
\hline & & & & Feelings about side effects & \\
\hline & & & & Satisfaction with therapy & \\
\hline \multirow{7}{*}{$\begin{array}{l}\text { Consumer Quality Index } \\
\text { Breast Care (CQI-BC) [37] }\end{array}$} & \multirow[t]{7}{*}{ Experiences } & \multirow{7}{*}{$\begin{array}{l}152 \text { ( } 118 \text { items related } \\
\text { to patients' experiences) }\end{array}$} & \multirow[t]{2}{*}{ Never, Sometimes, Usually, Always. } & \multirow{2}{*}{$\begin{array}{l}\text { Conduct of professionals during } \\
\text { breast examination }\end{array}$} & Emotional support \\
\hline & & & & & Information \& communication \\
\hline & & & \multirow[t]{2}{*}{ Yes, No. } & Conduct of general practitioner & Integrated \& coordinated care \\
\hline & & & & Conduct of nurses & Respectful to patients' values \\
\hline & & & \multirow{3}{*}{$\begin{array}{l}\text { A big problem, A small problem, } \\
\text { No problem. }\end{array}$} & Conduct of surgeon & \\
\hline & & & & Autonomy regarding treatment & \\
\hline & & & & $\begin{array}{l}\text { Autonomy regarding follow-up } \\
\text { treatment }\end{array}$ & \\
\hline
\end{tabular}

Never, Rarely, Sometimes, Frequently,

5 point scale from Not important (0) to Very important (4)

Very satisfied (4).

5 point scale with 1 representing the worst response and 5 representing 
Table 2 Measurement features of PROMs and included IOM-endorsed patient-centred care dimensions (Continued)

\begin{tabular}{|c|c|c|c|c|c|}
\hline & & & & $\begin{array}{l}\text { Conduct of professionals during } \\
\text { radiotherapy }\end{array}$ & \\
\hline & & & & Information on radiotherapy & \\
\hline & & & & $\begin{array}{l}\text { Conduct of professionals during } \\
\text { chemotherapy }\end{array}$ & \\
\hline & & & & Information on chemotherapy & \\
\hline & & & & Cooperation & \\
\hline & & & & Accessibility of care & \\
\hline & & & & Continuity psychosocial care & \\
\hline & & & & Continuity physiotherapy & \\
\hline & & & & Continuity rehabilitation & \\
\hline \multirow{15}{*}{$\begin{array}{l}\text { EORTC cancer in-patient } \\
\text { satisfaction with care } \\
\text { measure (EORTC } \\
\text { IN-PATSAT32) [38] }\end{array}$} & \multirow[t]{15}{*}{ Satisfaction } & \multirow[t]{15}{*}{32} & \multirow{15}{*}{$\begin{array}{l}\text { Poor, Fair, Good, Very good, } \\
\text { Excellent. }\end{array}$} & Doctors' technical skills & Emotional support \\
\hline & & & & Doctors' interpersonal skills & Information \& communication \\
\hline & & & & Doctors' information provision & Integrated \& coordinated care \\
\hline & & & & Doctors' availability & Physical comfort \\
\hline & & & & Nurses' technical skills & Respectful to patients' values \\
\hline & & & & Nurses' interpersonal skills & \\
\hline & & & & Nurses' information provision & \\
\hline & & & & Nurses' availability & \\
\hline & & & & Exchange of information & \\
\hline & & & & $\begin{array}{l}\text { Other hospital staff interpersonal } \\
\text { skills and information provision }\end{array}$ & \\
\hline & & & & Waiting time & \\
\hline & & & & Hospital access & \\
\hline & & & & Comfort & \\
\hline & & & & General & \\
\hline & & & & satisfaction & \\
\hline \multirow{3}{*}{$\begin{array}{l}\text { Indicators (Head \& Neck } \\
\text { Cancer) [39] }\end{array}$} & \multirow[t]{3}{*}{ Experiences } & \multirow{3}{*}{$\begin{array}{l}23 \text { specific indicators } \\
\text { for patients }\end{array}$} & \multirow[t]{3}{*}{ - } & Patient-oriented quality of care & Emotional support \\
\hline & & & & Organisational quality of care & Information \& communication \\
\hline & & & & Medical/technical quality of care & Integrated \& coordinated care \\
\hline \multirow{6}{*}{$\begin{array}{l}\text { Indicators (Non-small Cell } \\
\text { Lung Cancer) [40] }\end{array}$} & \multirow[t]{6}{*}{ Experiences } & \multirow[t]{6}{*}{56} & \multirow{4}{*}{$\begin{array}{l}1=\text { Not done, } 2=\text { Done, but } \\
\text { inadequately, } 3 \text { = Done adequately, } \\
4=\text { Done excellently. }\end{array}$} & Access & Emotional support \\
\hline & & & & Follow up & Family \& friends \\
\hline & & & & Communication and respect & Information \& communication \\
\hline & & & & Patient \& family involvement & Integrated \& coordinated care \\
\hline & & & \multirow[t]{2}{*}{ Yes, No } & Information & Physical comfort \\
\hline & & & & Coordination & Respectful to patients' values \\
\hline
\end{tabular}

satisfaction with care

measure (EORTC

IN-PATSAT32) [38]
$1=$ Not done, $2=$ Done, but
inadequately, $3=$ Done adequately,
$4=$ Done excellently.

Yes, No radiotherapy

Conduct of professionals during

Cooperation

continuity rehabilitatio

Doctors' interpersonal skil ss

Doctors' information provision

Nurses' information provision

Exchange of information

ther hospital staff interperson

Waiting time

Hospital access

satisfaction

Access

Coordination 
Table 2 Measurement features of PROMs and included IOM-endorsed patient-centred care dimensions (Continued)

\begin{tabular}{|c|c|c|c|c|c|}
\hline & & & & Physical support & \\
\hline & & & & Emotional \& psychosocial support & \\
\hline \multirow{3}{*}{$\begin{array}{l}\text { Medical Care Questionnaire } \\
\text { (MCQ) [41] }\end{array}$} & \multirow[t]{3}{*}{ Experiences } & \multirow[t]{3}{*}{15} & \multirow[t]{3}{*}{-} & Communication & Emotional support \\
\hline & & & & Preferences & Integrated \& coordinated care \\
\hline & & & & Coordination & Respectful to patients' values \\
\hline \multirow{4}{*}{$\begin{array}{l}\text { Modified Version of the } \\
\text { Perceived Involvement } \\
\text { in Care Scale (M-PICS) [42] }\end{array}$} & \multirow[t]{4}{*}{ Experiences } & \multirow[t]{4}{*}{20} & \multirow[t]{4}{*}{$1=$ All the time to $5=$ Never. } & Health care provider information & Information \& communication \\
\hline & & & & Patient information & \multirow[t]{3}{*}{ Respectful to patients' values } \\
\hline & & & & Patient decision making & \\
\hline & & & & Health care provider facilitation & \\
\hline \multirow{5}{*}{$\begin{array}{l}\text { Oncology Patients' } \\
\text { Perceptions of the } \\
\text { Quality of Nursing Care } \\
\text { Scale (OPPQNCS) [43] }\end{array}$} & \multirow[t]{5}{*}{ Experiences } & \multirow{5}{*}{$\begin{array}{l}40 \text { (and 18-item short } \\
\text { form created) }\end{array}$} & \multirow{5}{*}{$\begin{array}{l}1=\text { Never to } 6=\text { Always, Didn't matter, } \\
\text { Don't know. }\end{array}$} & Responsiveness & Emotional support \\
\hline & & & & Individualization & Family \& friends \\
\hline & & & & Coordination & Information \& communication \\
\hline & & & & Proficiency & Integrated \& coordinated care \\
\hline & & & & & Respectful to patients' values \\
\hline \multirow{8}{*}{$\begin{array}{l}\text { Pain Care Quality Survey } \\
\text { (PainCQ) }[44,45]\end{array}$} & \multirow[t]{8}{*}{ Experiences } & \multirow[t]{8}{*}{33} & \multirow{8}{*}{$\begin{array}{l}1=\text { Strongly disagree to } 6=\text { Strongly } \\
\text { agree. }\end{array}$} & PainCQ-Interdisciplinary scale: & Family \& friends \\
\hline & & & & Partnership with healthcare team & Information \& communication \\
\hline & & & & Comprehensive interdisciplinary & Integrated \& coordinated care \\
\hline & & & & pain care & Physical comfort \\
\hline & & & & PainCQ-Nursing scale: & Respectful to patients' values \\
\hline & & & & Being treated right & \\
\hline & & & & Comprehensive nursing pain care & \\
\hline & & & & Efficacy of pain management & \\
\hline \multirow[t]{6}{*}{ PASQOC questionnaire [46] } & \multirow[t]{6}{*}{ Satisfaction } & \multirow[t]{6}{*}{120} & \multirow{6}{*}{$\begin{array}{l}\text { Nominal or ordinal scales and some } \\
\text { interval scales }\end{array}$} & 5 dimensions reported & Information \& communication \\
\hline & & & & Patient-provider relationship & Respectful to patients' values \\
\hline & & & & Premises & \\
\hline & & & & Information on diagnosis \& treatment & \\
\hline & & & & Information on treatment consequences & \\
\hline & & & & Relationship between patient \& nurse & \\
\hline \multirow{3}{*}{$\begin{array}{l}\text { Patient Satisfaction with } \\
\text { Cancer Care [47] }\end{array}$} & \multirow[t]{3}{*}{ Satisfaction } & \multirow[t]{3}{*}{18} & \multirow{3}{*}{$\begin{array}{l}1=\text { Strongly Agree to } 5=\text { Strongly } \\
\text { Disagree }\end{array}$} & \multirow{3}{*}{$\begin{array}{l}1 \text { component structure - satisfaction } \\
\text { with cancer care }\end{array}$} & Information \& communication \\
\hline & & & & & Integrated \& coordinated care \\
\hline & & & & & Respectful to patients' values \\
\hline \multirow{4}{*}{$\begin{array}{l}\text { Perceived Physician's } \\
\text { Communication Style } \\
\text { Scale [48] }\end{array}$} & \multirow[t]{4}{*}{ Experiences } & \multirow[t]{4}{*}{27} & $1=$ Strongly Disagree, $3=$ Neutral, & Acceptive & Emotional support \\
\hline & & & & Patient-centered & Information \& communication \\
\hline & & & & Attentive & Respectful to patients' values \\
\hline & & & & Facilitative & \\
\hline
\end{tabular}


Table 2 Measurement features of PROMs and included IOM-endorsed patient-centred care dimensions (Continued)

\begin{tabular}{|c|c|c|c|c|c|}
\hline \multirow{22}{*}{$\begin{array}{l}\text { Prostate Care Questionnaire } \\
\text { for Patients (PCQ-P) [49] }\end{array}$} & \multirow[t]{22}{*}{ Experiences } & \multirow[t]{22}{*}{106 (Sections A-E). } & \multirow{22}{*}{$\begin{array}{l}\text { Various scales - please see } \\
\text { reference [63] }\end{array}$} & Information from additional file 1 & Information \& communication \\
\hline & & & & Section A: GP visits and referral & Integrated \& coordinated care \\
\hline & & & & Explanation & Physical comfort \\
\hline & & & & Experience of referral & Respectful to patients' values \\
\hline & & & & Taking the problem seriously & \\
\hline & & & & Section B: Tests at the hospital & \\
\hline & & & & Explanation \& support & \\
\hline & & & & Quality of care & \\
\hline & & & & Appointment & \\
\hline & & & & $\begin{array}{l}\text { Section C: Diagnosis and treatment } \\
\text { decision }\end{array}$ & \\
\hline & & & & Explanation \& support & \\
\hline & & & & Making treatment decision & \\
\hline & & & & Getting the diagnosis & \\
\hline & & & & Length of wait & \\
\hline & & & & Section D: Treatment and discharge & \\
\hline & & & & Preparation for discharge & \\
\hline & & & & Treatment & \\
\hline & & & & Information & \\
\hline & & & & Section E: Monitoring & \\
\hline & & & & Explanation \& reassurance & \\
\hline & & & & Advice & \\
\hline & & & & Choice & \\
\hline \multirow[t]{8}{*}{ QUOTE Breast Cancer $[50,51]$} & \multirow[t]{8}{*}{ Experiences } & \multirow[t]{8}{*}{33} & Performance: & \multirow{2}{*}{$\begin{array}{l}\text { Patient education regarding aspects } \\
\text { related to postoperative treatment }\end{array}$} & Information \& communication \\
\hline & & & \multirow[t]{2}{*}{ Never, Sometimes, Usually, Always. } & & Integrated \& coordinated care \\
\hline & & & & Services by the breast nurse & Respectful to patients' values \\
\hline & & & \multirow[t]{2}{*}{ Yes, No. } & Services by the surgeon & \\
\hline & & & & $\begin{array}{l}\text { Patient education regarding activities } \\
\text { at home }\end{array}$ & \\
\hline & & & $\begin{array}{l}\text { Not applicable/l do not know added } \\
\text { to a subset of items. }\end{array}$ & $\begin{array}{l}\text { Patient education regarding aspects } \\
\text { related to preoperative treatment }\end{array}$ & \\
\hline & & & Importance: & & \\
\hline & & & $\begin{array}{l}\text { Not important, Fairly important, } \\
\text { Important, Extremely important. }\end{array}$ & & \\
\hline \multirow[t]{2}{*}{ QUOTE chemo $_{[52]}$} & \multirow[t]{2}{*}{ Experiences } & \multirow[t]{2}{*}{67} & Performance: & Treatment-related information & Emotional support \\
\hline & & & Yes, No. & Prognosis information & Family \& friends \\
\hline
\end{tabular}


Table 2 Measurement features of PROMs and included IOM-endorsed patient-centred care dimensions (Continued)

\begin{tabular}{|c|c|c|c|c|c|}
\hline & & & Importance: & Rehabilitation information & Information \& communication \\
\hline & & & & Coping information & Physical comfort \\
\hline & & & Not important, Fairly important, & Interpersonal communication & Respectful to patients' values \\
\hline & & & Important, very important. & Tailored communication & \\
\hline & & & & Affective communication & \\
\hline \multirow[t]{13}{*}{ REPERES-60 [53] } & \multirow[t]{13}{*}{ Experiences } & \multirow[t]{13}{*}{60} & Bad, Fair, Good, Very good, Excellent. & Access to primary care & Emotional support \\
\hline & & & & Access to secondary care & Information \& communication \\
\hline & & & $\begin{array}{l}\text { Completely agree, Agree generally, } \\
\text { No marked opinion, Do not really }\end{array}$ & $\begin{array}{l}\text { Competence and communication } \\
\text { skills of primary care doctors }\end{array}$ & Integrated \& coordinated care \\
\hline & & & & $\begin{array}{l}\text { Competence of secondary care } \\
\text { doctors }\end{array}$ & Respectful to patients' values \\
\hline & & & & $\begin{array}{l}\text { Communication skills of secondary } \\
\text { care doctors }\end{array}$ & \\
\hline & & & $\begin{array}{l}\text { Bad, Fair, Good, Very good, Excellent, } \\
\text { Not concerned. }\end{array}$ & Choice among different doctors & \\
\hline & & & & Human qualities of doctors & \\
\hline & & & & Global satisfaction & \\
\hline & & & & Cover for medical expenses & \\
\hline & & & & $\begin{array}{l}\text { Listening abilities and information } \\
\text { provided by doctors }\end{array}$ & \\
\hline & & & & $\begin{array}{l}\text { Organisation and follow-up of } \\
\text { medical care provision }\end{array}$ & \\
\hline & & & & Psychological support & \\
\hline & & & & Material environment & \\
\hline \multirow{2}{*}{$\begin{array}{l}\text { Satisfaction with Cancer } \\
\text { Information Profile } \\
\text { (SCIP) [54] }\end{array}$} & \multirow[t]{2}{*}{ Satisfaction } & 21 & $\begin{array}{l}\text { Too much, About right, Too little, } \\
\text { None wanted. }\end{array}$ & $\begin{array}{l}\text { Satisfaction with the amount and } \\
\text { content of information }\end{array}$ & Information \& communication \\
\hline & & & $\begin{array}{l}\text { Very satisfied, Satisfied, Neither, } \\
\text { Dissatisfied, Very dissatisfied. }\end{array}$ & $\begin{array}{l}\text { Satisfaction with the form and } \\
\text { timing of the information received }\end{array}$ & \\
\hline \multirow[t]{6}{*}{ SAT-RAR [55] } & \multirow[t]{6}{*}{ Experiences } & \multirow[t]{6}{*}{23} & Poor, Fair, Good, Very good, & \multirow{2}{*}{$\begin{array}{l}\text { Perception of the radiotherapist or } \\
\text { radiotherapy technicians }\end{array}$} & Emotional support \\
\hline & & & xcellent. & & Information \& communication \\
\hline & & & Not at all, A little, Quite a bit, Very & Global satisfaction & Physical comfort \\
\hline & & & & \multirow[t]{3}{*}{ Treatment experience } & \\
\hline & & & $\begin{array}{l}\text { Poor, Moderate, Good, Very good, } \\
\text { Excellent. }\end{array}$ & & \\
\hline & & & $\begin{array}{l}\text { Disagree, Unsure, Tend to agree, } \\
\text { Agree, Strongly agree. }\end{array}$ & & \\
\hline
\end{tabular}

Bad, Fair, Good, Very good, Excellent, ot concerned.

Agree, Strongly agree. 
Table 3 IOM patient-centred care dimensions captured by PROMs

\begin{tabular}{|c|c|c|c|c|c|c|}
\hline \multirow[t]{2}{*}{ Measure } & \multicolumn{6}{|c|}{ IOM patient-centred care dimensions } \\
\hline & $\begin{array}{c}\text { Emotional } \\
\text { support }\end{array}$ & $\begin{array}{l}\text { Family \& } \\
\text { friends }\end{array}$ & $\begin{array}{l}\text { Information \& } \\
\text { communication }\end{array}$ & $\begin{array}{l}\text { Integrated \& } \\
\text { coordinated care }\end{array}$ & $\begin{array}{l}\text { Physical } \\
\text { comfort }\end{array}$ & $\begin{array}{l}\text { Respectful to } \\
\text { patients' values }\end{array}$ \\
\hline APECC [32] & $\sqrt{ }$ & & $\sqrt{ }$ & $\sqrt{ }$ & & $\sqrt{ }$ \\
\hline $\begin{array}{l}\text { Cancer Care Coordination Questionnaire for } \\
\text { Patients [33] }\end{array}$ & $\sqrt{ }$ & $\sqrt{ }$ & $\sqrt{ }$ & $\sqrt{ }$ & & \\
\hline $\begin{array}{l}\text { Cancer Patient Information Importance and } \\
\text { Satisfaction Tool [34] }\end{array}$ & $\sqrt{ }$ & & $\sqrt{ }$ & & $\sqrt{ }$ & \\
\hline CTSQ $[35,36]$ & & & & & & $\sqrt{ }$ \\
\hline CQI-BC [37] & $\sqrt{ }$ & & $\sqrt{ }$ & $\sqrt{ }$ & & $\sqrt{ }$ \\
\hline EORTC IN-PATSAT32 [38] & $\sqrt{ }$ & & $\sqrt{ }$ & $\sqrt{ }$ & $\sqrt{ }$ & $\sqrt{ }$ \\
\hline Indicators (Head \& Neck Cancer) [39] & $\sqrt{ }$ & & $\sqrt{ }$ & $\sqrt{ }$ & & \\
\hline Indicators (Non-small Cell Lung Cancer) [40] & $\sqrt{ }$ & $\sqrt{ }$ & $\sqrt{ }$ & $\sqrt{ }$ & $\sqrt{ }$ & $\sqrt{ }$ \\
\hline MCQ [41] & $\sqrt{ }$ & & & $\sqrt{ }$ & & $\sqrt{ }$ \\
\hline M-PICS [42] & & & $\sqrt{ }$ & & & $\sqrt{ }$ \\
\hline OPPQNCS [43] & $\sqrt{ }$ & $\sqrt{ }$ & $\sqrt{ }$ & $\sqrt{ }$ & & $\sqrt{ }$ \\
\hline PainCQ $[44,45]$ & & $\sqrt{ }$ & $\sqrt{ }$ & $\sqrt{ }$ & $\sqrt{ }$ & $\sqrt{ }$ \\
\hline PASQOC questionnaire [46] & & & $\sqrt{ }$ & & & $\sqrt{ }$ \\
\hline Patient Satisfaction with Cancer Care [47] & & & $\sqrt{ }$ & $\sqrt{ }$ & & $\sqrt{ }$ \\
\hline $\begin{array}{l}\text { Perceived Physician's Communication Style } \\
\text { Scale [48] }\end{array}$ & $\sqrt{ }$ & & $\sqrt{ }$ & & & $\sqrt{ }$ \\
\hline PCQ-P [49] & & & $\sqrt{ }$ & $\sqrt{ }$ & $\sqrt{ }$ & $\sqrt{ }$ \\
\hline QUOTE Breast Cancer [50,51] & & & $\sqrt{ }$ & $\sqrt{ }$ & & $\sqrt{ }$ \\
\hline QUOTE chemo [52] & $\sqrt{ }$ & $\sqrt{ }$ & $\sqrt{ }$ & & $\sqrt{ }$ & $\sqrt{ }$ \\
\hline REPERES-60 [53] & $\sqrt{ }$ & & $\sqrt{ }$ & $\sqrt{ }$ & & $\sqrt{ }$ \\
\hline SCIP [54] & & & $\sqrt{ }$ & & & \\
\hline SAT-RAR [55] & $\sqrt{ }$ & & $\sqrt{ }$ & & $\sqrt{ }$ & \\
\hline
\end{tabular}

\section{Construct validity}

Eighteen measures met the criteria for adequate construct validity [32,33,35,37,38,41-44,46-50,52-55]. Sixteen measures conducted factor analyses [32,33,35, $37,41-44,46-50,52,53,55]$ (although only seven reported eigenvalues) $[33,41,44,47,48,50,53]$, nine measures examined convergent validity $(r>0.40)$ or divergent validity $(\mathrm{r}<0.30)$ with existing instruments $[35,38,42$, $46,47,49,52-54]$ and six measures demonstrated significant differences on scores between known groups $[35,38,41,42,53,55]$.

\section{Cross-cultural adaptation}

Three measures were re-validated with non-English speaking populations. The EORTC IN-PATSAT32 was validated with Sri Lankan cancer patients [56]; the Modified version of the Perceived Involvement in Care Scale (M-PICS) was validated with Lithuanian cancer patients [57]; and the Oncology Patients' Perceptions of the Quality Nursing Care Scale (OPPQNCS) was validated with Turkish cancer patients [58].
Table 5 summarises which PROMs met the psychometric criteria considered adequate, as described above.

\section{Psychometric properties of PROMs containing all six IOM patient-centred care dimensions}

The Indicators (Non-small Cell Lung Cancer) measure [40] was the only PROM that contained items covering all six IOM dimensions of patient-centred care. This measure met the criteria considered adequate for face/ content validity, but not for any other psychometric criteria evaluated in this review.

\section{Discussion}

This is the first review to identify how many of the six IOM-endorsed dimensions of patient-centred care [1] are covered in existing PROMs assessing the quality of cancer care. Our findings demonstrate that since the publication of the IOM's Crossing the Quality Chasm report in 2001 [1], only one of 21 patient-centred cancer care instruments, the Indicators (Non-small Cell Lung Cancer) measure, included questions relating to the six 
Table 4 Psychometric properties of PROMs assessing quality of patient-centred cancer care

\begin{tabular}{|c|c|c|c|c|c|c|c|}
\hline \multirow[t]{2}{*}{ Measure } & \multirow[t]{2}{*}{ Face validity/content validity } & \multicolumn{3}{|c|}{ Construct validity } & \multirow{2}{*}{$\begin{array}{l}\text { Internal } \\
\text { consistency }\end{array}$} & \multirow{2}{*}{$\begin{array}{l}\text { Test-retest } \\
\text { reliability }\end{array}$} & \multirow{2}{*}{$\begin{array}{c}\text { Cross- } \\
\text { cultural } \\
\text { adaptation }\end{array}$} \\
\hline & & Factor analysis & Known groups & Existing measure & & & \\
\hline \multirow{11}{*}{$\begin{array}{l}\text { Assessment of Patient } \\
\text { Experiences of Cancer } \\
\text { Care (APECC) [32] }\end{array}$} & \multirow{11}{*}{$\begin{array}{l}\text { All items underwent cognitive } \\
\text { testing with nine cancer } \\
\text { survivors to ensure that the } \\
\text { questions and response options } \\
\text { were understandable and } \\
\text { related to the concept being } \\
\text { measured. }\end{array}$} & \multirow{11}{*}{$\begin{array}{l}\text { Confirmatory factor analysis } \\
\text { indicated a reasonably good fit } \\
\text { for the } 10 \text {-factor model (com- } \\
\text { parative fit index }=0.93 \text { ). }\end{array}$} & \multirow[t]{11}{*}{-} & \multirow[t]{11}{*}{-} & $\begin{array}{l}\text { Getting needed } \\
\text { care: } a=.76\end{array}$ & \multirow[t]{11}{*}{-} & \multirow[t]{11}{*}{-} \\
\hline & & & & & $\begin{array}{l}\text { Timeliness of } \\
\text { care: } a=.62\end{array}$ & & \\
\hline & & & & & $\begin{array}{l}\text { Waiting time in } \\
\text { physician's office: } \\
a=.65\end{array}$ & & \\
\hline & & & & & $\begin{array}{l}\text { Information } \\
\text { exchange: } \alpha=.92\end{array}$ & & \\
\hline & & & & & $\begin{array}{l}\text { Physicians' } \\
\text { affective } \\
\text { behavior: } \mathrm{a}=.92\end{array}$ & & \\
\hline & & & & & $\begin{array}{l}\text { Physicians' } \\
\text { knowledge: } \mathbf{a} \\
=.86\end{array}$ & & \\
\hline & & & & & $\begin{array}{l}\text { Interaction with } \\
\text { nurses: } \mathrm{a}=.82\end{array}$ & & \\
\hline & & & & & $\begin{array}{l}\text { Interaction with } \\
\text { office staff: a } \\
=.90\end{array}$ & & \\
\hline & & & & & $\begin{array}{l}\text { Health } \\
\text { promotion: } \mathbf{a} \\
=.88\end{array}$ & & \\
\hline & & & & & $\begin{array}{l}\text { Coordination of } \\
\text { care: N/A }\end{array}$ & & \\
\hline & & & & & $\begin{array}{l}\text { Overall rating of } \\
\text { care: } \mathrm{a}=.87\end{array}$ & & \\
\hline \multirow[t]{3}{*}{$\begin{array}{l}\text { Cancer Care Coordination } \\
\text { Questionnaire for Patients [33] }\end{array}$} & \multirow{3}{*}{$\begin{array}{l}\text { Literature review undertaken to } \\
\text { identify relevant issues and } \\
\text { existing instruments and focus } \\
\text { groups and semi-structured in- } \\
\text { terviews with } 24 \text { cancer patients } \\
\text { and carers and } 29 \text { clinicians. } \\
\text { Draft questionnaire was } \\
\text { reviewed by clinicians and re- } \\
\text { searchers to assess face validity } \\
\text { and clarity of wording. }\end{array}$} & $\begin{array}{l}\text { Exploratory factor analysis. } \\
\text { Principal factor method followed } \\
\text { by a promax rotation. }\end{array}$ & \multirow[t]{3}{*}{-} & \multirow[t]{3}{*}{-} & \multirow{3}{*}{$\begin{array}{l}\text { Total scale: } \mathrm{a}=.88 \\
\text { Communication: } \\
a=.87 \\
\text { Navigation: } \\
a=.73\end{array}$} & $\begin{array}{l}\text { Sample 1: } 119 \\
\text { patients completed } \\
\text { the survey twice - } \\
\text { mailed } 2 \text { weeks } \\
\text { after receipt of first } \\
\text { survey. }\end{array}$ & \multirow[t]{3}{*}{-} \\
\hline & & $\begin{array}{l}\text { Factor loadings }>.40 \text { with the } \\
\text { exception of one item }(0.37) \text {. }\end{array}$ & & & & \multirow{2}{*}{$\begin{array}{l}\text { Kappa for individual } \\
\text { items ranged from } \\
0.29 \text { to } 0.69 \text {. Four } \\
\text { items with values } \\
\text { less than } 0.40 \text { were } \\
\text { eliminated. }\end{array}$} & \\
\hline & & Eigenvalues $>1$ & & & & & \\
\hline $\begin{array}{l}\text { Cancer Patient Information } \\
\text { Importance and Satisfaction } \\
\text { Tool [34] }\end{array}$ & $\begin{array}{l}\text { Literature review and extensive } \\
\text { qualitative interviews with } \\
\text { cancer patients. Tool was field- }\end{array}$ & - & - & - & $\begin{array}{l}\text { Information } \\
\text { importance: } \\
a=89\end{array}$ & - & - \\
\hline
\end{tabular}


Table 4 Psychometric properties of PROMs assessing quality of patient-centred cancer care (Continued)

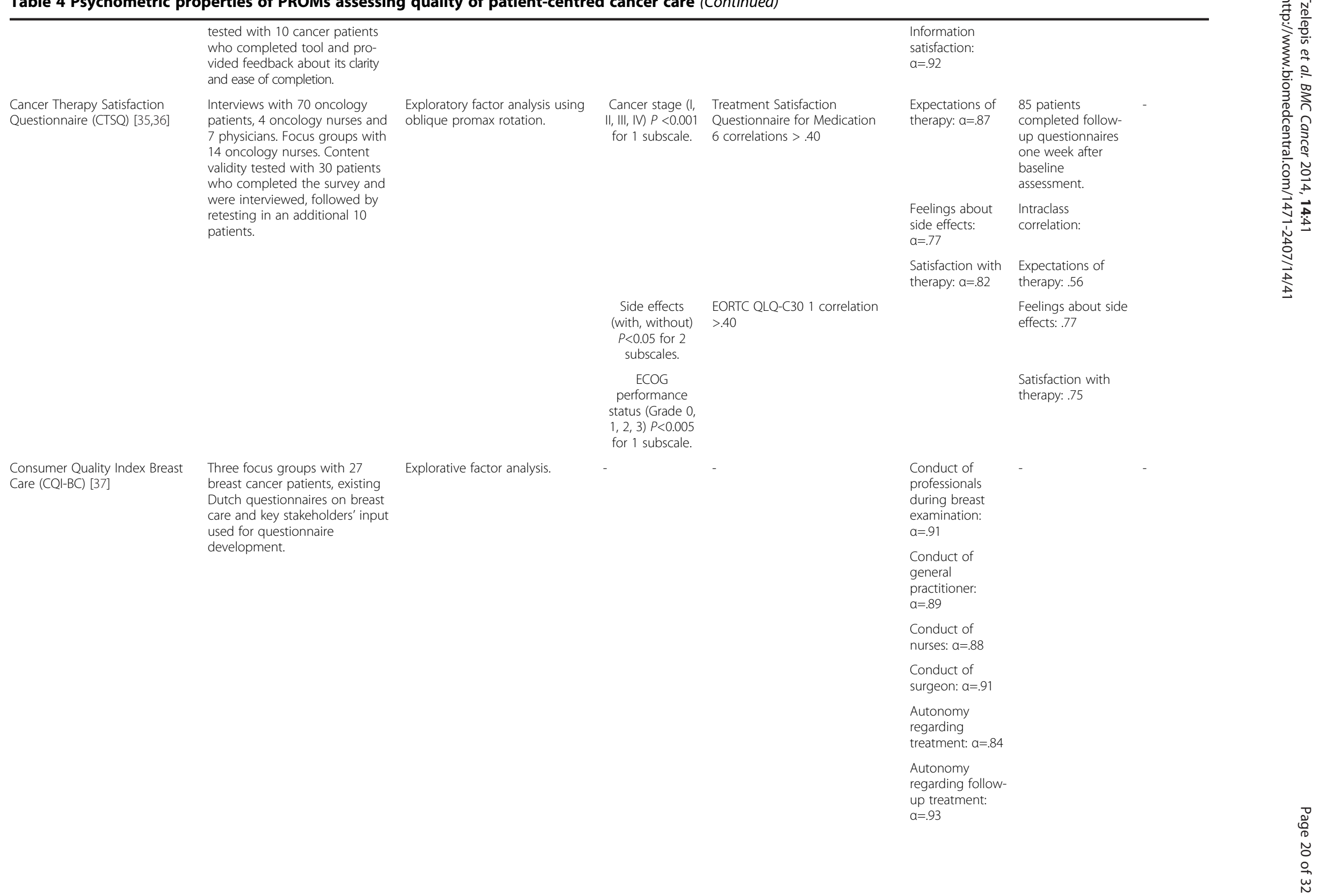


EORTC cancer in-patient satisfac- Adapted from existing patient tion with care measure (EORTC IN-PATSAT32) [38] satisfaction questionnaires

(Comprehensive Assessment of

Satisfaction with Care and

EORTC QLQ-SAT32) [64,65], as

well as interviews with oncology

specialists and cancer patients.
Conduct of

professionals

during

radiotherapy:

$a=.89$

Information on

radiotherapy:

$\mathrm{a}=89$

Conduct of

professionals

during

chemotherapy:

$a=.90$

Information on

chemotherapy:

$a=.85$

Cooperation:

$a=.88$

Accessibility of

care: $\mathrm{a}=.68$

Continuity

psychosocial

care: $\mathrm{a}=.83$

Continuity

physiotherapy:

$a=.82$

Continuity
rehabilitation:

$\mathrm{a}=80$

Age (less than 57 Oberst Patients' Perception of Doctors'

years, 57 years or Care Quality and Satisfaction technical skills:

more) $P<0.05$

for 4 subscales.

Doctors'

interpersonal

skills: $\mathrm{a}=.91-.94$

113 patients Validated

recruited from one with Sri

centre for follow-up Lankan

approximately 2 cancer

than compulsory,

post-compulsory)

weeks after first

cancer

post-compulsory)
$P<0.05$ for 2

assessment.

Doctors'

information

provision: $\mathrm{a}=.90$

$-.94$

Doctors'

availability: $\mathrm{a}=.86$

$-.91$

Nurses' technical

skills: $\alpha=.90-.94$ 


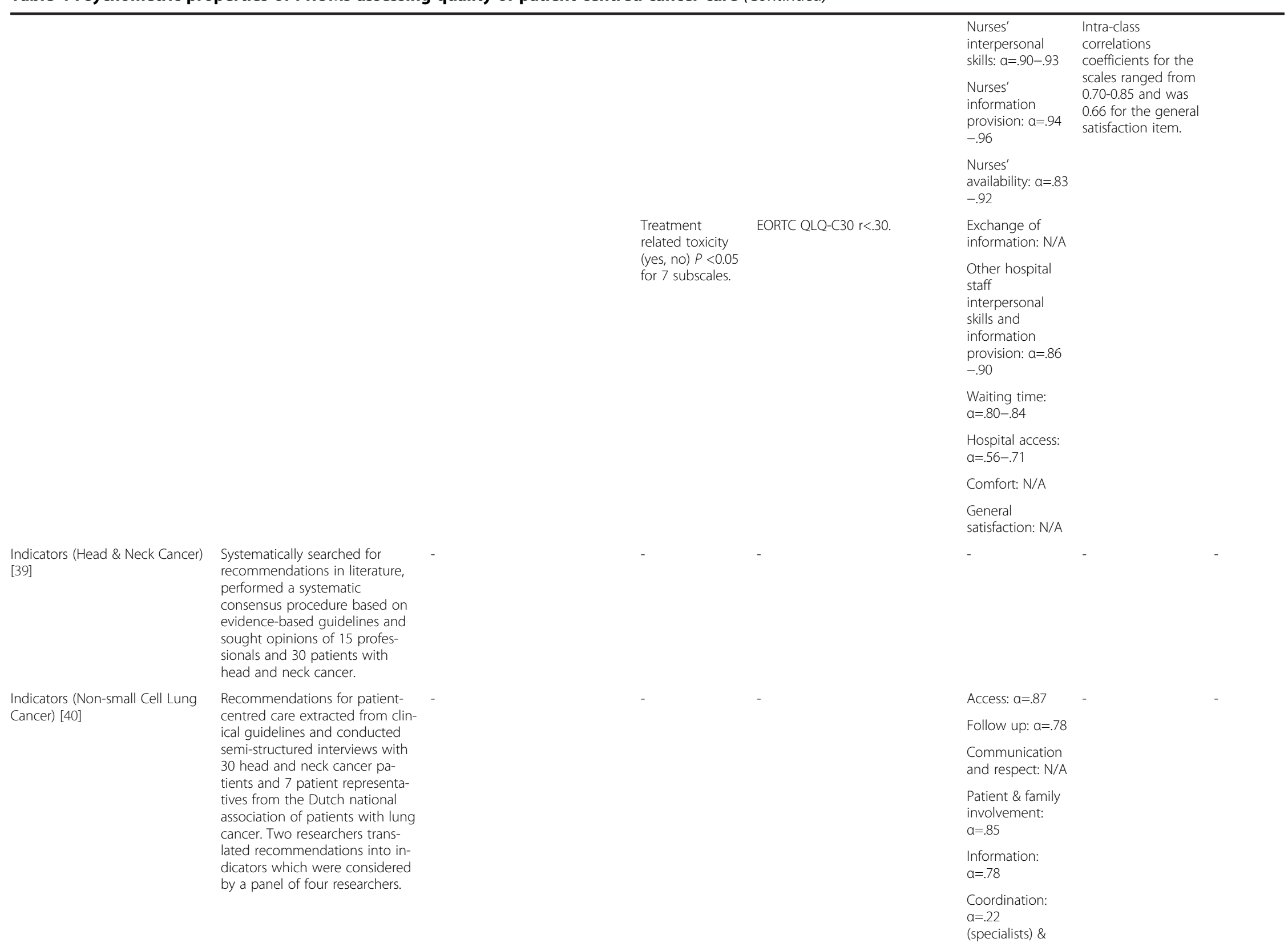




\begin{tabular}{|c|c|c|c|c|c|c|c|}
\hline & & & & & $\begin{array}{l}a=.68 \text { (oncology } \\
\text { nurses) }\end{array}$ & & \\
\hline & & & & & $\begin{array}{l}\text { Physical support: } \\
\text { N/A }\end{array}$ & & \\
\hline & & & & & $\begin{array}{l}\text { Emotional \& } \\
\text { psychosocial } \\
\text { support: } \alpha=.67\end{array}$ & & \\
\hline \multirow[t]{3}{*}{$\begin{array}{l}\text { Medical Care Questionnaire } \\
\text { (MCQ) [41] }\end{array}$} & \multirow{3}{*}{$\begin{array}{l}\text { Literature review of existing } \\
\text { instruments, modification (items } \\
\text { removed, reworded, generated) } \\
\text { of an existing instrument by an } \\
\text { expert panel ( } 3 \text { medical } \\
\text { oncologists and one oncologist } \\
\text { in training) using a consensus } \\
\text { procedure, instrument } \\
\text { administered to } 200 \text { oncology } \\
\text { outpatients and then refined. }\end{array}$} & $\begin{array}{l}\text { Exploratory factor analysis using } \\
\text { oblique rotation (Phase } 3 \text { ) and } \\
\text { confirmatory factor analysis } \\
\text { (Phase 4). }\end{array}$ & \multirow{3}{*}{$\begin{array}{l}\text { Cancer type } \\
\text { (Breast, } \\
\text { Genitourinary, } \\
\text { Gynecological, } \\
\text { Melanoma, } \\
\text { Sarcoma, Other) } \\
\text { P }<0.05 \text { for } 3 \\
\text { subscales. }\end{array}$} & \multirow[t]{3}{*}{-} & $\begin{array}{l}\text { Communication: } \\
a=.69\end{array}$ & \multirow[t]{3}{*}{ - } & \multirow[t]{3}{*}{-} \\
\hline & & Factor loadings $>.40$ & & & $\begin{array}{l}\text { Preferences: } \\
\mathrm{a}=.84\end{array}$ & & \\
\hline & & Eigenvalues > 1 & & & $\begin{array}{l}\text { Coordination: } \\
a=.75\end{array}$ & & \\
\hline \multirow[t]{10}{*}{$\begin{array}{l}\text { Modified Version of the } \\
\text { Perceived Involvement in Care } \\
\text { Scale (M-PICS) [42] }\end{array}$} & \multirow{10}{*}{$\begin{array}{l}\text { Literature review and } \\
\text { consultation with pain clinicians } \\
\text { guided augmentation and } \\
\text { addition of items on the original } \\
\text { Perceived Involvement in Care } \\
\text { Scale (PICS). }\end{array}$} & $\begin{array}{l}\text { Exploratory factor analysis - } \\
\text { principal components analysis } \\
\text { with oblique rotation. }\end{array}$ & $\begin{array}{l}\text { Age } P<0.01 \text { for } 1 \\
\text { subscale. }\end{array}$ & Barriers Questionnaire-II & $\begin{array}{l}\text { Total scale: } \mathrm{a}= \\
.87\end{array}$ & - & \multirow{10}{*}{$\begin{array}{l}\text { Validated } \\
\text { with } \\
\text { Lithuanian } \\
\text { cancer } \\
\text { patients } \\
(\mathrm{n}=30)[57]\end{array}$} \\
\hline & & Factor loadings $>.40$ & $\begin{array}{l}\text { Ethnicity (Latina; } \\
\text { Caucasian/ } \\
\text { African- } \\
\text { American) }\end{array}$ & 2 correlations $>.40$ & $\begin{array}{l}\text { Health care } \\
\text { provider } \\
\text { information: } a= \\
.90\end{array}$ & & \\
\hline & & & $\begin{array}{l}P<0.01 \text { for } 2 \\
\text { subscales. }\end{array}$ & Mental Health Inventory & $\begin{array}{l}\text { Patient } \\
\text { information: } \mathrm{a}= \\
.82\end{array}$ & & \\
\hline & & & & 3 correlations $<.30$ & $\begin{array}{l}\text { Patient decision } \\
\text { making: } \mathrm{a}=.80\end{array}$ & & \\
\hline & & & & $\begin{array}{l}\text { Medical Outcomes Study Short- } \\
\text { Form 12: Mental Component } \\
\text { Scale }\end{array}$ & \multirow{6}{*}{$\begin{array}{l}\text { Health care } \\
\text { provider } \\
\text { facilitation: } \mathrm{a}= \\
.80\end{array}$} & & \\
\hline & & & & 3 correlations $<.30$ & & & \\
\hline & & & & $\begin{array}{l}\text { Medical Outcomes Study Short- } \\
\text { Form 12: Physical Component } \\
\text { Scale }\end{array}$ & & & \\
\hline & & & & 4 correlations $<.30$ & & & \\
\hline & & & & $\begin{array}{l}\text { Patient Satisfaction } \\
\text { Questionnaire }\end{array}$ & & & \\
\hline & & & & 3 correlations $>.40$ & & & \\
\hline \multirow[t]{2}{*}{$\begin{array}{l}\text { Oncology Patients' Perceptions } \\
\text { of the Quality of Nursing Care } \\
\text { Scale (OPPQNCS) [43] }\end{array}$} & \multirow{2}{*}{$\begin{array}{l}\text { Interviews with cancer patients } \\
\text { about their perceptions of } \\
\text { nursing care, items and } \\
\text { subscales generated from this } \\
\text { qualitative work, an expert } \\
\text { methods consultant evaluated }\end{array}$} & \multirow[t]{2}{*}{$\begin{array}{l}\text { Exploratory factor analysis - } \\
\text { principal components analyses } \\
\text { with promax (oblique) rotation. }\end{array}$} & & \multirow[t]{2}{*}{-} & $\begin{array}{l}\text { Total scale: } \mathrm{a}= \\
.99\end{array}$ & & \multirow{2}{*}{$\begin{array}{l}\text { Validated } \\
\text { with } \\
\text { Turkish } \\
\text { cancer } \\
\text { patients } \\
(n=54) \text { [58] }\end{array}$} \\
\hline & & & & & $\begin{array}{l}\text { Responsiveness: } \\
a=.99\end{array}$ & & \\
\hline
\end{tabular}


Table 4 Psychometric properties of PROMs assessing quality of patient-centred cancer care (Continued)

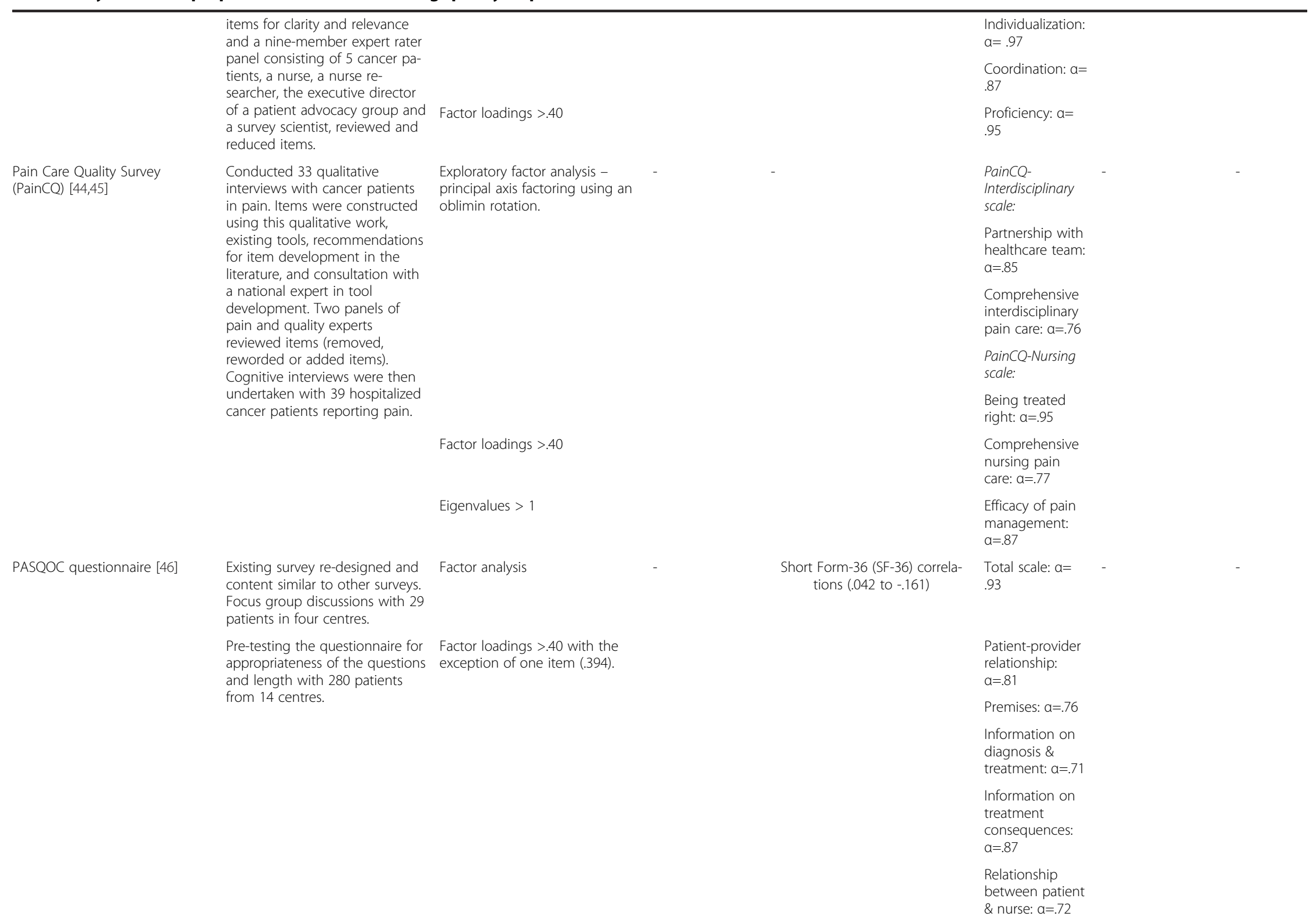


Table 4 Psychometric properties of PROMs assessing quality of patient-centred cancer care (Continued)

\begin{tabular}{|c|c|c|c|c|c|c|c|}
\hline \multirow[t]{3}{*}{$\begin{array}{l}\text { Patient Satisfaction with Cancer } \\
\text { Care [47] }\end{array}$} & \multirow{3}{*}{$\begin{array}{l}\text { Item pool based on literature } \\
\text { review and existing measures, } \\
\text { expert feedback, group } \\
\text { discussion and consensus. }\end{array}$} & $\begin{array}{l}\text { Factor analysis - principal } \\
\text { components analysis }\end{array}$ & \multirow[t]{3}{*}{-} & \multirow{3}{*}{$\begin{array}{l}\text { Communication and Attitudinal } \\
\text { Self-Efficacy - Cancer } 1 \text { correl- } \\
\text { ation }>.40 \text { (sample 2). }\end{array}$} & \multirow{3}{*}{$\begin{array}{l}\text { Total scale: } \mathrm{a}= \\
.95 \text { and } .96 \text { (for } \\
\text { two samples). }\end{array}$} & \multirow[t]{3}{*}{-} & \multirow[t]{3}{*}{-} \\
\hline & & Factor loadings $>.40$ & & & & & \\
\hline & & Eigenvalue $>1$ & & & & & \\
\hline \multirow[t]{5}{*}{$\begin{array}{l}\text { Perceived Physician's } \\
\text { Communication Style Scale [48] }\end{array}$} & \multirow{5}{*}{$\begin{array}{l}\text { Initial item pool created from } \\
\text { literature review and } 25 \text { cancer } \\
\text { patients' opinions about the } \\
\text { physician's communication } \\
\text { style. } 114 \text { nurses assessed the } \\
\text { content validity of items. Items } \\
\text { pre-tested with } 70 \text { breast cancer } \\
\text { patients. }\end{array}$} & $\begin{array}{l}\text { Factor analysis using varimax } \\
\text { procedure. }\end{array}$ & \multirow[t]{5}{*}{-} & \multirow[t]{5}{*}{-} & $\begin{array}{l}\text { Total scale: } \mathrm{a}= \\
.95\end{array}$ & \multirow[t]{5}{*}{-} & \multirow[t]{5}{*}{-} \\
\hline & & Factor loadings $>.40$ & & & Acceptive: $\mathrm{a}=.90$ & & \\
\hline & & \multirow[t]{3}{*}{ Eigenvalues > 1} & & & $\begin{array}{l}\text { Patient-centered: } \\
\mathrm{a}=.90\end{array}$ & & \\
\hline & & & & & Attentive: $\mathrm{a}=.73$ & & \\
\hline & & & & & Facilitative: $a=.76$ & & \\
\hline \multirow[t]{11}{*}{$\begin{array}{l}\text { Prostate Care Questionnaire for } \\
\text { Patients (PCQ-P) [49] }\end{array}$} & \multirow{11}{*}{$\begin{array}{l}\text { Initial items developed through a } \\
\text { literature review and interviews } \\
\text { with patients and service providers. } \\
\text { Semi-structured interviews with } 20 \\
\text { prostate cancer patients who com- } \\
\text { pleted survey. }\end{array}$} & $\begin{array}{l}\text { Exploratory principal } \\
\text { components analysis with } \\
\text { varimax rotation. }\end{array}$ & \multirow[t]{11}{*}{-} & $\begin{array}{l}\text { National Centre for Social } \\
\text { Research Shortened } \\
\text { Questionnaire }\end{array}$ & Section $A: a=.80$ & \multirow{4}{*}{$\begin{array}{l}148(50 \%) \text { patients } \\
\text { from two hospitals } \\
\text { completed retest } \\
\text { survey mailed } 3 \\
\text { weeks later. }\end{array}$} & - \\
\hline & & \multirow{10}{*}{$\begin{array}{l}\text { Factor loadings for each section } \\
\text { of } 0.3 \text { and higher presented in } \\
\text { an additional file. }\end{array}$} & & Sections B \& C & Section $B: a=.63$ & & \\
\hline & & & & $r<.30$ & Section C: $a=.77$ & & \\
\hline & & & & Sections D \& E r $>.40$ & Section $\mathrm{D}: \mathrm{a}=.80$ & & \\
\hline & & & & & Section $\mathrm{E}: \mathrm{a}=.68$ & $\begin{array}{l}\text { Intraclass } \\
\text { correlation } \\
\text { coefficient: }\end{array}$ & \\
\hline & & & & & & Section A: .68 & \\
\hline & & & & & & Section B: .57 & \\
\hline & & & & & & Section C: .61 & \\
\hline & & & & & & Section D: .73 & \\
\hline & & & & & & Section E: .70 & \\
\hline & & & & & & $\begin{array}{l}\text { Identical responses } \\
\text { to individual } \\
\text { questions: } 52.6 \% \text { to } \\
100 \%\end{array}$ & \\
\hline \multirow[t]{4}{*}{ QUOTE Breast Cancer [50,51] } & \multirow{4}{*}{$\begin{array}{l}\text { Based on eight focus groups } \\
\text { with } 72 \text { breast cancer patients } \\
\text { and concept mapping sessions } \\
\text { with } 67 \text { breast cancer patients a } \\
\text { pilot questionnaire was } \\
\text { developed. Two researchers } \\
\text { categorised and reduced the } \\
\text { aspects of care identified. }\end{array}$} & $\begin{array}{l}\text { Exploratory factor analysis (i.e., } \\
\text { principal axis factoring) - } \\
\text { oblique rotation. }\end{array}$ & \multirow[t]{2}{*}{$\begin{array}{l}\text { Age }(18-49,50- \\
65,>65 \text { years }) \text { no } \\
\text { difference. }\end{array}$} & - & $\begin{array}{l}\text { Patient } \\
\text { education } \\
\text { regarding } \\
\text { aspects related } \\
\text { to postoperative } \\
\text { treatment: } \alpha=.83\end{array}$ & \multirow[t]{4}{*}{-} & - \\
\hline & & $\begin{array}{l}\text { Factor loadings }>.40 \text { except for } \\
0.35 \text { loading and } 6 \text { separate } \\
\text { items. }\end{array}$ & & & $\begin{array}{l}\text { Services by the } \\
\text { breast nurse: } \\
a=.89\end{array}$ & & \\
\hline & & \multirow[t]{2}{*}{ Eigenvalues >1 } & \multirow{2}{*}{$\begin{array}{l}\text { Education } \\
\text { (primary school, } \\
\text { secondary } \\
\text { school, college/ }\end{array}$} & & $\begin{array}{l}\text { Services by the } \\
\text { surgeon: } \mathrm{a}=.85\end{array}$ & & \\
\hline & & & & & $\begin{array}{l}\text { Patient } \\
\text { education }\end{array}$ & & \\
\hline
\end{tabular}


Table 4 Psychometric properties of PROMs assessing quality of patient-centred cancer care (Continued)

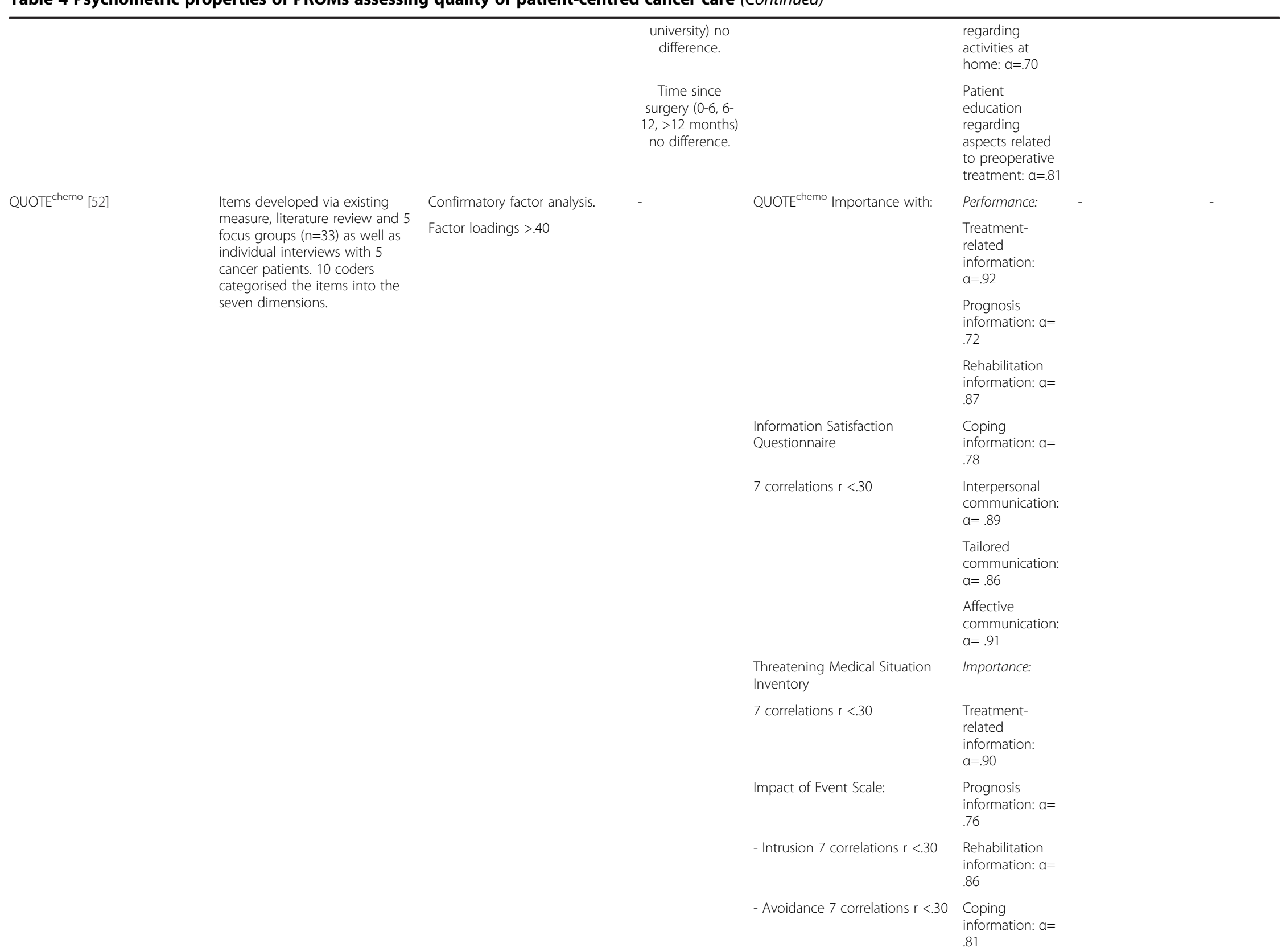




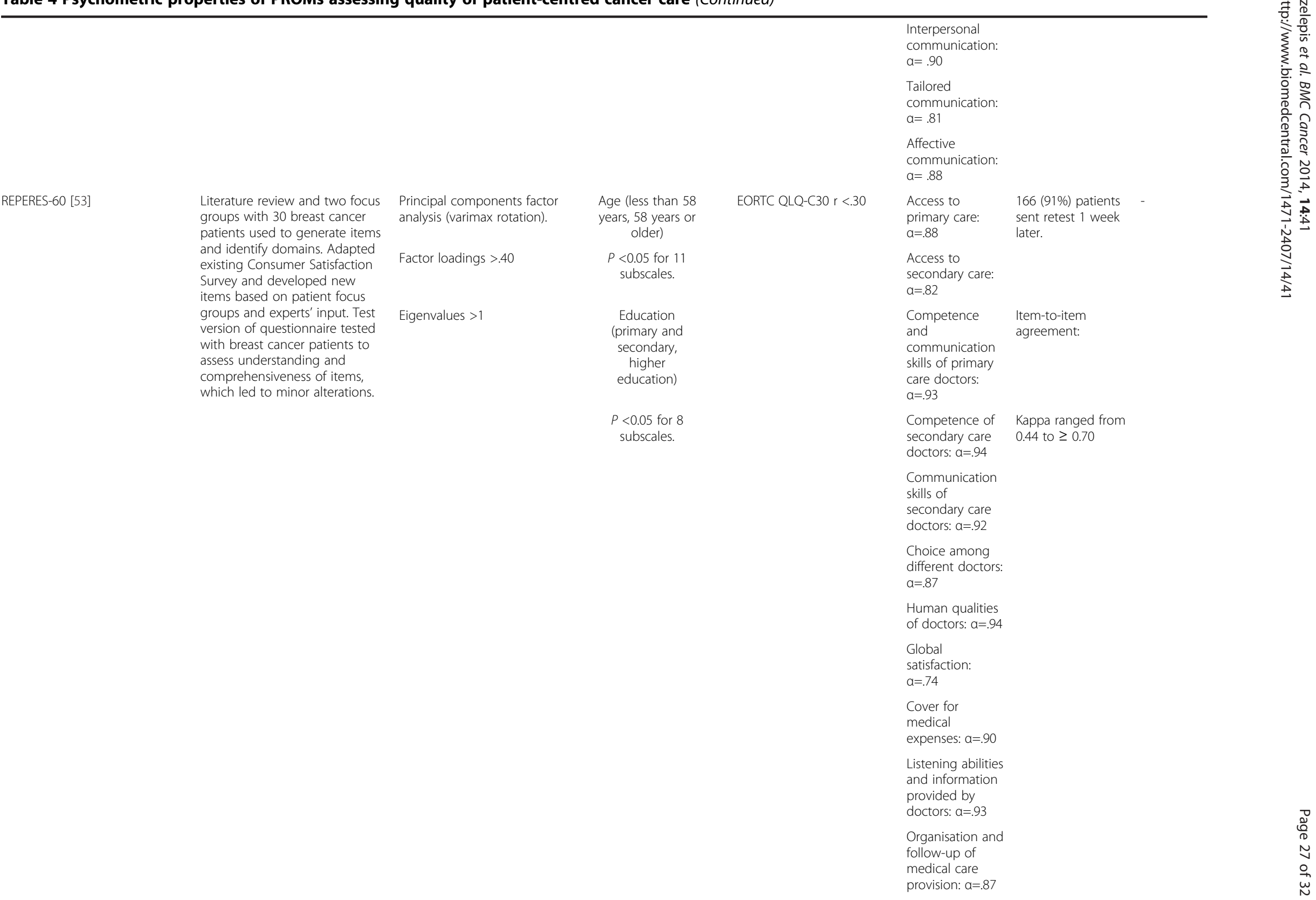


Satisfaction with Cancer

Information Profile (SCIP) [54

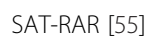

SAT-RAR [55]

Review of the literature,

Exploratory factorial analysis with selection and formation of items varimax rotation followed by based on relevant themes and a Confirmatory factorial analysis. cancer care satisfaction

questionnaire, pilot-testing of

preliminary questionnaire with

10 patients to evaluate rele-

pance, content validity and com-

prehensibility of items, survey

reviewed by clinicians in the study.

Most factor loadings $>.40$, except 3 factor loadings $>.30$
Psychological

support: $\alpha=.88$

Material

environment:

$a=.89$

IIIness Perception QuestionnaireRevised

Satisfaction with the amount and

content of

information:

$\mathrm{a}=.89$

Satisfaction with

the form and

timing of

$\mathrm{a}=.87$

Total scale: $\mathrm{a}=.86$

ast high schoo

completed, high

school not

completed)

$P<0.01$ in 1

subscale.

Perception of

the

radiotherapist or

radiotherapy

$a=94$

Marital status

married, no

Global

satisfaction:

$\mathrm{a}=.80$

$P<0.01$ in 1

subscale.

Work status

(employed, no

employed)

$P<0.01$ in 2

subscales.

Type of

radiotherapy

(respiratory

gating, no

gating, no

respiratory

$P<0.01$ in 2

subscales. 
Table 5 PROMs demonstrating adequate psychometric properties based on recommended criteria

\begin{tabular}{|c|c|c|c|c|c|c|c|}
\hline \multirow[t]{2}{*}{ Measure } & \multirow{2}{*}{$\begin{array}{l}\text { Face/content } \\
\text { validity }\end{array}$} & \multicolumn{3}{|c|}{ Construct validity } & \multirow{2}{*}{$\begin{array}{l}\text { Internal } \\
\text { consistency }\end{array}$} & \multirow{2}{*}{$\begin{array}{l}\text { Test-retest } \\
\text { reliability }\end{array}$} & \multirow{2}{*}{$\begin{array}{l}\text { Cross-cultural } \\
\text { adaptation }\end{array}$} \\
\hline & & $\begin{array}{l}\text { Factor } \\
\text { analysis }\end{array}$ & $\begin{array}{l}\text { Known } \\
\text { groups }\end{array}$ & $\begin{array}{l}\text { Existing } \\
\text { measure }\end{array}$ & & & \\
\hline APECC [32] & & $\sqrt{ }$ & & & & & \\
\hline $\begin{array}{l}\text { Cancer Care Coordination Questionnaire for } \\
\text { Patients [33] }\end{array}$ & $\sqrt{ }$ & $\sqrt{ }$ & & & $\sqrt{ }$ & & \\
\hline \multicolumn{8}{|l|}{$\begin{array}{l}\text { Cancer Patient Information Importance and } \\
\text { Satisfaction Tool [34] }\end{array}$} \\
\hline CTSQ $[35,36]$ & $\sqrt{ }$ & $\sqrt{ }$ & $\sqrt{ }$ & $\sqrt{ }$ & & & \\
\hline CQI-BC [37] & $\sqrt{ }$ & $\sqrt{ }$ & & & & & \\
\hline EORTC IN-PATSAT32 [38] & $\sqrt{ }$ & & $\sqrt{ }$ & $\sqrt{ }$ & & & $\sqrt{ }$ \\
\hline Indicators (Head \& Neck Cancer) [39] & $\sqrt{ }$ & & & & & & \\
\hline Indicators (Non-small Cell Lung Cancer) [40] & $\sqrt{ }$ & & & & & & \\
\hline MCQ [41] & $\sqrt{ }$ & $\sqrt{ }$ & $\sqrt{ }$ & & & & \\
\hline M-PICS [42] & & $\sqrt{ }$ & $\sqrt{ }$ & $\sqrt{ }$ & $\sqrt{ }$ & & $\sqrt{ }$ \\
\hline OPPQNCS [43] & $\sqrt{ }$ & $\sqrt{ }$ & & & $\sqrt{ }$ & & $\sqrt{ }$ \\
\hline PainCQ $[44,45]$ & $\sqrt{ }$ & $\sqrt{ }$ & & & & & \\
\hline PASQOC questionnaire [46] & & $\sqrt{ }$ & & $\sqrt{ }$ & $\sqrt{ }$ & & \\
\hline Patient Satisfaction with Cancer Care [47] & & $\sqrt{ }$ & & $\sqrt{ }$ & $\sqrt{ }$ & & \\
\hline $\begin{array}{l}\text { Perceived Physician's Communication Style } \\
\text { Scale [48] }\end{array}$ & $\sqrt{ }$ & $\sqrt{ }$ & & & $\sqrt{ }$ & & \\
\hline PCQ-P [49] & $\sqrt{ }$ & $\sqrt{ }$ & & $\sqrt{ }$ & & & \\
\hline QUOTE Breast Cancer $[50,51]$ & $\sqrt{ }$ & $\sqrt{ }$ & & & & & \\
\hline QUOTE chemo $[52]$ & $\sqrt{ }$ & $\sqrt{ }$ & & $\sqrt{ }$ & & & \\
\hline REPERES-60 [53] & $\sqrt{ }$ & $\sqrt{ }$ & $\sqrt{ }$ & $\sqrt{ }$ & & & \\
\hline SCIP [54] & & & & $\sqrt{ }$ & & & \\
\hline SAT-RAR [55] & $\sqrt{ }$ & $\sqrt{ }$ & $\sqrt{ }$ & & $\sqrt{ }$ & & \\
\hline
\end{tabular}

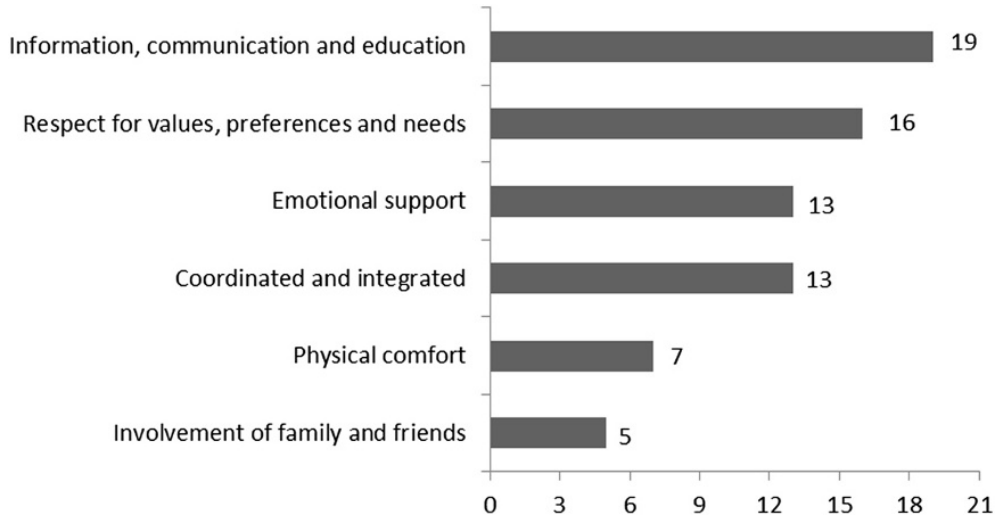

Figure 2 Frequency of IOM-endorsed patient-centred care dimensions across 21 measures. 
IOM dimensions of patient-centred care [40]. However this measure only met the criteria considered acceptable for face/content validity. Further psychometric testing of the Indicators (Non-small Cell Lung Cancer) measure is required before more definitive conclusions can be drawn about its reliability and validity.

Across measures, the most commonly included patientcentred care dimensions were "information, communication and education" (19 of 21 measures) followed by "respectful to patients' values, preferences, and expressed needs" (16 of 21 measures). In contrast, only seven measures examined patient's perceptions of "physical comfort" and five assessed the "involvement and wellbeing of family and friends." Possible explanations for the lesser focus on issues related to family and friends could include: 1) researchers/health professionals perceiving issues related to information and communication as the most important features of patient-centredness; 2) that the patients and survey developers involved in item selection only wished to focus on specific aspects of care; and 3) issues related to family and friends are considered a less crucial feature of cancer care. Furthermore, the measures may not have adequately captured the IOM's six dimensions of patient-centred care because they were not developed for that purpose. For example, a measure's objective may have been to focus solely or primarily on physical comfort, rather than to address the IOM's six dimensions of patient-centred care. Nevertheless, the lack of PROMs that included all six IOM dimensions of patient-centred care [1] limits the potential of these existing measures to capture the whole-person orientation of health care and is likely to result in an incomplete representation of the quality of care provided to cancer patients.

Improvements to the reliability of existing patientcentred care PROMs and better reporting of their internal consistency, are needed. Only seven of the 21 measures met the criteria considered adequate for internal consistency by reporting a Cronbach's alpha $>0.70$ for the total scale and each sub-scale [33,42,43,46-48,55]. A further six measures showed that all subscales had a Cronbach's alpha $>0.70[34,35,44,52-54]$, but failed to report the internal consistency for the total scale. However, interpretation of internal consistency findings should always consider that when a subscale has a large number of items, Cronbach's alpha can be artificially high $[59,60]$. Test-retest validity was very rarely considered during the development of PROMs assessing patient-centred cancer care. Although four of the five measures that examined test-retest reliability administered a second survey within 2-14 days $[33,35,38,53]$, none of the measures demonstrated acceptable agreement between scores for the total scale, subscales and items across the two administrations $[18,19]$. However possible explanations for the lack of adequate test-retest reliability among PROMs assessing patient-centred cancer care may include that: 1) patients' experiences of care, particularly for those receiving active treatment, actually changed between the initial and second administration of the measure; and 2) completing the initial measure altered patients' expectations of patient-centred care and as a result patients rated their care differently during the second administration of the measure. Nonetheless, future research that develops PROMs of patient-centred cancer care, or validates existing measures should examine test-retest reliability, with the aim of achieving high item-to-item agreement. Item-to-item agreement is necessary [21], as high agreement between overall subscale scores can be obtained even when corresponding items within the subscale are answered differently across the two administrations.

In terms of the validity of the PROMs developed to assess patient-centred care, most of the measures met the criteria considered adequate for face/content validity (15 of 21 measures) and construct validity (18 of 21 measures). Factor analysis was the most common strategy adopted to measure construct validity (16 measures), however, few studies indicated whether eigenvalues $>1$ [24] were achieved $[33,41,44,47,48,50,53]$. Eigenvalues are used to determine the number of subscales within the measure by applying the eigenvalues $>1$ rule which produces psychometrically reliable and psychologically meaningful results [24]. Thus improvements to reporting whether eigenvalues were $>1$ appears necessary for PROMs that examine patient-centred cancer care.

The context in which these PROMs assessed patientcentred cancer care should be considered. Most measures were developed with cancer patients recruited from hospitals or treatment centres [33,34,38-44,46-50,53-55]. Only one measure was developed with patients recruited via a population-based cancer registry [32], despite benefits of such recruitment including the ability to sample a representative group of patients at different stages of the disease and with varied experiences of cancer care [61]. Although measuring the quality of patient-centred cancer care during initial treatment and hospital visits is crucial, undertaking such assessments with cancer survivors who no longer visit the hospital regularly is also important. For instance, women diagnosed with breast cancer have reported that the quality and duration of their follow-up consultations with clinicians had declined compared to the quality and duration of their initial treatment experiences [62].

The limitations of this review include that studies available in a non-English language peer-reviewed journal and the grey literature were excluded which could have led to some bias in the findings. Furthermore, the survey developers' reasons for constructing the PROM should be considered. It is possible that the PROM's objective may have been to focus on specific features of patient-centred care rather than to include items that covered the IOM's six dimensions of patient-centred care. This may explain why most PROMs did not adequately address 
the IOM's six dimensions of patient-centred care. Additionally, insufficient or unavailable reporting of the 21 PROMs' psychometric properties may have influenced the ratings regarding the adequacy of the measure's psychometric properties. We did not contact the authors of each PROM to enquire if additional unpublished psychometric information was available for that measure.

\section{Conclusions}

Quality improvements to the health care system can be guided by PROMs assessing the quality of patient-centred cancer care. The Indicators (Non-small Cell Lung Cancer) measure [40] was the only identified PROM that included questions relating to the six IOM endorsed dimensions of patient-centred care [1], however psychometric inadequacies and/or incomplete reporting indicates that further psychometric testing of this measure is required. Using more than one measure or further developing existing measures to include all six patient-centred care dimensions could improve the assessment and the delivery of patient-centred care. Additionally, given the lack of psychometrically rigorous PROMs developed to assess patient-centred cancer care that capture the six IOM dimensions, the construction of new comprehensive measures whose psychometric properties are adequate may also be warranted.

\section{Abbreviations}

IOM: Institute of Medicine; PROMs: Patient-reported outcome measures.

\section{Competing interests}

The authors declare that they have no competing interests.

\section{Authors' contributions}

FT, SKR, RWSF, TCM, MLC and CLP were involved in study conception and design of the systematic review. FT and SKR undertook literature searches, coded the studies for eligibility and evaluated and extracted information from eligible studies. FT drafted the manuscript. All the authors revised the article critically and approved the final version of the manuscript.

\section{Acknowledgements \\ This research was undertaken by the Priority Research Centre for Health Behaviour at the University of Newcastle which receives infrastructure support from the Hunter Medical Research Institute. Dr Flora Tzelepis was supported by a Leukaemia Foundation of Australia and Cure Cancer Australia Foundation Post-Doctoral Research Fellowship. Dr Tara Clinton-McHarg was supported by a Leukaemia Foundation Post-Doctoral Research Fellowship. These funding bodies did not have any role in the study design, collection, analysis and interpretation of data, in the writing of the manuscript and in the decision to submit the manuscript for publication.}

Received: 10 August 2013 Accepted: 20 January 2014

Published: 25 January 2014

\section{References}

1. Institute of Medicine: Crossing The Quality Chasm: A New Health System for the 21st Century. Washington DC: National Academy Press; 2001.

2. Gerteis M, Edgman-Levitan S, Daley J: Through the Patient's Eyes. Understanding and Promoting Patient-centered Care. San Francisco, CA: Jossey-Bass; 1993.

3. Hayman JA: Measuring the quality of care in radiation oncology. Semin Radiat Oncol 2008, 18:201-206.

4. Fink R: Pain assessment: the cornerstone to optimal pain management. BUMC Proceedings 2000, 13:236-239.
5. Wong WS, Fielding R: The association between patient satisfaction and quality of life in Chinese lung and liver cancer patients. Med Care 2008, 46:293-302.

6. Von Essen L, Larsson G, Oberg K, Sjoden PO: 'Satisfaction with care': associations with health-related quality of life and psychosocial function among Swedish patients with endocrine gastrointestinal tumours. Eur J Cancer Care 2002, 11:91-99.

7. Kim S, Bae J-M, Kim Y-W, Ryu KW, Lee JH, Noh J-H, Sohn T-S, Homg S-K, Lee MK, Park SM, Yun YH: Self-reported experience and outcomes of care among stomach cancer patients at a median follow-up time of 27 months from diagnosis. Support Care Cancer 2008, 16:831-839.

8. Frojd C, Lampic C, Larsson G, von Essen L: Is satisfaction with doctors' care related to health-related quality of life, anxiety and depression among patients with carcinoid tumours? A longitudinal report. Scand J Caring Sci 2009, 23:107-116.

9. Mager WM, Andrykowski MA: Communication in the cancer 'bad news' consultation: patient perceptions and psychological adjustment. Psychooncology 2002, 11:35-46.

10. Bickell NA, Weidmann J, Fei K, Lin JJ, Leventhal H: Underuse of breast cancer adjuvant treatment: patient knowledge, beliefs, and medical mistrust. J Clin Oncol 2009, 27:5160-5167.

11. Kahn KL, Schneider EC, Malin JL, Adams JL, Epstein AM: Patient centered experiences in breast cancer: predicting long-term adherence to tamoxifen use. Med Care 2007, 45:431-439.

12. Mandelblatt JS, Sheppard VB, Hurria A, Kimmick G, Isaacs C, Taylor KL, et al: Breast cancer adjuvant chemotherapy decisions in older women: the role of patient preference and interactions with physicians. J Clin Oncol 2010, 28:3146-3153.

13. Shin DW, Park JH, Shim EJ, Hahm Ml, Park EC: Predictors and outcomes of feeling of insufficient consultation time in cancer care in Korea: results of a nationwide multicenter survey. Support Care Cancer 2012, 20:1965-1973.

14. Kowalski C, Nitzsche A, Scheibler F, Steffen P, Albert U-S, Pfaff H: Breast cancer patients' trust in physicians: the impact of patients' perception of physicians' communication behaviors and hospital organizational climate. Patient Educ Couns 2009, 77:344-348.

15. Crow R, Gage H, Hampson S, Hart J, Kimber A, Storey L, Thomas H: The measurement of satisfaction with healthcare: implications for practice from a systematic review of the literature. Health Technol Assess 2002, 6:1-244.

16. Cleary PD, Edgman-Levitan S: Health care quality. Incorporating consumer perspectives. JAMA 1997, 278:1608-1612.

17. Mitchell PH, Heinrich J, Moritz P, Hinshaw AS: Measurement into practice. Summary and recommendations. Med Care 1997, 35(Suppl 11):NS124-127.

18. Lohr KN, Aaronson NK, Alonso J, Burnam MA, Patrick DL, Perrin EB, Roberts JS: Evaluating quality-of-life and health status instruments: development of scientific review criteria. Clin Ther 1996, 18:979-992.

19. McDowell I: Measuring Health: A Guide to Rating Scales and Questionnaires. New York: Oxford University Press; 2006.

20. Marx RG, Menezes A, Horovitz $L$, Jones EC, Warren RF: A comparison of two time intervals for test-retest reliability of health status instruments. $J$ Clin Epidemiol 2003, 56:730-735.

21. Viswanathan M: Measurement Error and Research Design. CA: Sage Publications; 2005.

22. Anastasi A, Urbina S: Psychological Testing. Prentice Hall: Upper Saddle River, NJ: 1997.

23. Cohen J: Statistical Power Analysis for the Behavioural Sciences. Hillsdale, NJ: Erlbaum; 1988.

24. Kaiser HF: The application of electronic computers to factor analysis. Educ Psychol Meas 1960, 20:141-151.

25. Castle NG, Brown J, Hepner KA, Hays RD: Review of the literature on survey instruments used to collect data on hospital patients' perceptions of care. Health Serv Res 2005, 40:1996-2017.

26. Rubin HR: Patient evaluations of hospital care. A review of the literature. Med Care 1990, 28(Suppl 9):S3-9.

27. Bredart A, Sultan S, Regnault A: Patient satisfaction instruments for cancer clinical research or practice. Expert Rev 2010, 10:129-141.

28. Hudon C, Fortin M, Haggerty $\lrcorner$, Lambert M, Poitras ME: Measuring patients' perceptions of patient-centered care: a systematic review of tools for family medicine. Ann Fam Med 2011, 9:155-164.

29. Hearn J, Higginson IJ: Outcome measures in palliative care for advanced cancer patients: a review. J Public Health Med 1997, 19:193-199. 
30. Clinton-McHarg T, Carey M, Sanson-Fisher R, Shakeshaft A, Rainbird K: Measuring the psychosocial health of adolescent and young adult (AYA) cancer survivors: a critical review. Health Qual Life Outcomes 2010, 8:25.

31. Poinsot R, Altmeyer A, Conroy T, Savignoni A, Asselain B, Léonard I, Marx E, Cosquer M, Sévellec M, Gledhill J: Multisite validation study of questionnaire assessing out-patient satisfaction with care questionnaire in ambulatory chemotherapy or radiotherapy treatment. Bull Cancer 2006, 93:315-327.

32. Arora NK, Reeve BB, Hays RD, Clauser SB, Oakley-Girvan I: Assessment of quality of cancer-related follow-up care from the cancer survivor's perspective. J Clin Oncol 2011, 29:1280-1289.

33. Young JM, Walsh J, Butow PN, Solomon MJ, Shaw J: Measuring cancer care coordination: development and validation of a questionnaire for patients. BMC Cancer 2011, 11:298.

34. Fitch F, McAndrew A: A performance measurement tool for cancer patient information and satisfaction. J Cancer Educ 2011, 26:612-618.

35. Trask P, Tellefsen C, Epspindle D, Getter C, Hsu M: Psychometric validation of the Cancer Therapy Satisfaction Questionnaire. Value Health 2008, 11:669-679.

36. Abetz L, Coombs JH, Keininger DL, Earle CC, Wade C, Bury-Maynard D, Copley-Merriman K, Hsu M-A: Development of the cancer therapy satisfaction questionnaire: item generation and content validity testing Value Health 2005, 8(Suppl 1):S41-53.

37. Damman OC, Hendriks M, Sixma HJ: Towards more patient centred healthcare: a new Consumer Quality Index instrument to assess patients' experiences with breast care. Eur J Cancer 2009, 45:1569-1577.

38. Bredart A, Bottomley A, Blazeby JM, Conroy T, Coens C, D'Haese S, et al: An international prospective study of the EORTC cancer in-patient satisfaction with care measure (EORTC IN-PATSAT32). Eur J Cancer 2005, 41:2120-2131.

39. Ouwens MM, Marres HA, Hermens RR, Hulscher MM, van den Hoogen FJ, Grol RP, Wollersheim HC: Quality of integrated care for patients with head and neck cancer: development and measurement of clinical indicators. Head Neck 2007, 29:378-386.

40. Ouwens M, Hermens R, Hulscher M, Vonk-Okhuijsen S, Tjan-Heijnen V, Termeer R, Marres H, Wollersheim H, Grol R: Development of indicators for patient-centred cancer care. Support Care Cancer 2010, 18:121-130.

41. Harley C, Adams J, Booth L, Selby P, Brown J, Velikova G: Patient experiences of continuity of cancer care: development of a new Medical Care Questionnaire (MCQ) for oncology outpatients. Value Health 2009, 12:1180-1186.

42. Smith MY, Winkel G, Egert J, Diaz-Wionczek M, DuHamel KN: Patient-physician communication in the context of persistent pain: validation of a Modified Version of the Patients' Perceived Involvement in Care Scale. J Pain Symptom Manage 2006, 32:71-81

43. Radwin L, Alster K, Rubin KM: Development and testing of the Oncology Patients' Perceptions of the Quality of Nursing Care Scale. Oncol Nurs Forum 2003, 30:283-290.

44. Beck SL, Towsley GL, Pett MA, Berry PH, Smith EL, Brant JM, Guo J-W: Initia psychometric properties of the Pain Care Quality Survey (PainCQ). J Pain 2010, 11:1311-1319.

45. Beck SL, Towsley GL, Berry PH, Brant JM, Smith EM: Measuring the quality of care related to pain management: a multiple-method approach to instrument development. Nurs Res 2010, 59:85-92

46. Kleeberg UR, Tews JT, Ruprecht T, Hoing M, Kuhlmann A, Runge C: Patient satisfaction and quality of life in cancer outpatients: results of the PASQOC study. Support Care Cancer 2005, 13:303-10.

47. Jean-Pierre P, Fiscella K, Freund KM, Clark J, Darnell J, Holden A, Post D, Patierno SR, Winters PC, Patient Navigation Research Program Group: Structural and reliability analysis of a patient satisfaction with cancer-related care measure: a multisite patient navigation research program study. Cancer 2011, 117:854-861.

48. Takayama T, Yamazaki Y, Katsumata N: Relationship between outpatients' perceptions of physicians' communication styles and patients' anxiety levels in a Japanese oncology setting. Soc Sci Med 2001, 53:1335-1350.

49. Tarrant C, Baker R, Colman AM, Sinfield P, Agarwal S, Mellon JK, Steward W, Kockelbergh $\mathrm{R}$ : The prostate care questionnaire for patients (PCQ-P): reliability, validity and acceptability. BMC Health Serv Res 2009, 9:199.

50. de Kok M, Sixma HJM, van der Weijden T, Kessels AGH, Dirksen CD, Spijkers KFJ, et al: A patient-centred instrument for assessment of quality of breast cancer care: results of a pilot questionnaire. Qual Saf Health Care 2010, 19:e40.
51. de Kok M, Scholte RW, Sixma HJ, van der Weijden T, Spijkers KF, van de Velde CJ, Roukema JA, ven der Ent FW, Bell AV, von Meyenfeldt MF: The patient's perspective of the quality of breast cancer care. The development of an instrument to measure quality of care through focus groups and concept mapping with breast cancer patients. Eur J Cancer 2007, 43:1257-1264.

52. van Weert JCM, Jansen J, de Bruijn GJ, Noordman J, van Dulmen S, Bensing JM: QUOTEchemo: a patient-centred instrument to measure quality of communication preceding chemotherapy treatment through the patient's eyes. Eur J Cancer 2009, 45:2967-2976.

53. Defossez G, Mathoulin-Pelissier S, Ingrand I, Gasquet I, Sifer-Riviere L, Ingrand $P$, Salamon $R$, Migeot $V$, the REPERES research network: Satisfaction with care among patients with non-metastatic breast cancer: development and first steps of validation of the REPERES-60 questionnaire. BMC Cancer 2007, 7:129.

54. Llewellyn C, Horne R, McGurk M, Weinman J: Development and preliminary validation of a new measure to assess satisfaction with information among head and neck cancer patients: the Satisfaction with Cancer Information Profile (SCIP). Head Neck 2005, 28:540-548.

55. Bredart A, Morvan E, Savignoni A, Giraud P, Respiratory Gated Radiotherapy Study Group S-R: Patient's perception of care quality during radiotherapy sessions using respiratory gating techniques: validation of a specific questionnaire. Cancer Invest 2011, 29:145-152

56. Jayasekara H, Rajapaksa L, Bredart A: Psychometric evaluation of the European Organization for Research and Treatment of Cancer in-patient satisfaction with care questionnaire ('Sinhala' version) for use in a SouthAsian setting. Int J Qual Health Care 2008, 20:221-226.

57. Jacobsen R, Samsanaviciene J, Liuabarskiene Z, Sciupokas A: Barriers to pain management among Lithuanian cancer patients. Pain Pract 2010, 10:145-157.

58. Can G, Akin S, Aydiner A, Ozdilli K, Durna Z: Evaluation of the effect of care given by nursing students on oncology patients' satisfaction. Eur $J$ Oncol Nurs 2008, 12:387-392.

59. Streiner D, Norman G: Health Measurement Scales: A Practical Guide to their Development and Use. New York: Oxford University Press, Fourth; 2008.

60. Terwee CB, Bot SD, de Boer MR, van der Windt DA, Knol DL, Dekker J, Bouter LM, de Vet HC: Quality criteria were proposed for measurement properties of health status questionnaires. J Clin Epidemiol 2007, 60:34-42.

61. Sanson-Fisher R, Carey M, Mackenzie L, Hill D, Campbell S, Turner D: Reducing inequities in cancer care: the role of cancer registries. Cancer 2009, 115:3597-3605.

62. Lawler S, Spathonis K, Masters J, Adams J, Eakin E: Follow-up care after breast cancer treatment: experiences and perceptions of service provision and provider interactions in rural Australian women. Support Care Cancer 2011, 19:1975-1982.

63. The Prostate Care Questionnaire for Patients (PCQ-P). http://winden.co.uk/ Surveys/Prostate\%20Disease/Appendix\%2023\%20Patient\%20v5.1\%2008-0208\%2020-02-08.pdf. Accessed 16 November 2012. September 2007.

64. Bredart A, Mignot V, Rousseau A, Dolbeault S, Beauloye N, Adam V, Elie C, Leonard I, Asselain B, Conroy T: Validation of the EORTC QLQ-SAT32 cancer inpatient satisfaction questionnaire by self- versus interviewassessment comparison. Patient Educ Couns 2004, 54:207-212.

65. Bredart A, Razavi D, Delvaux N, Goodman V, Farvacques C, Van Heer C: A comprehensive assessment of satisfaction with care for cancer patients. Support Care Cancer 1998, 6:518-523.

doi:10.1186/1471-2407-14-41

Cite this article as: Tzelepis et al:: Are we missing the Institute of Medicine's mark? A systematic review of patient-reported outcome measures assessing quality of patient-centred cancer care. BMC Cancer 2014 14:41. 